\title{
ITANS: Incremental Task and Network Scheduling for Time- Sensitive Networks
}

This paper was downloaded from TechRxiv (https://www.techrxiv.org).

LICENSE

CC BY 4.0

SUBMISSION DATE / POSTED DATE

$07-11-2021 / 09-11-2021$

CITATION

Arestova, Anna; Baron, Wojciech (2021): ITANS: Incremental Task and Network Scheduling for TimeSensitive Networks. TechRxiv. Preprint. https://doi.org/10.36227/techrxiv.16944958.v1

DOI

10.36227/techrxiv.16944958.v1 


\title{
ITANS: Incremental Task and Network Scheduling for Time-Sensitive Networks
}

\author{
Anna Arestova, Wojciech Baron, Kai-Steffen Hielscher, and Reinhard German
}

\begin{abstract}
The rapid development in information and communication technology confronts designers of real-time systems with new challenges that have arisen due to the increasing amount of data and an intensified interconnection of functions. This is e.g. driven by recent trends such as automated driving in the automotive field and digitization in factory automation. For distributed safety-critical systems, this progression has the impact that the complexity of scheduling tasks with precedence constraints organized in so-called task chains increases the more data has to be exchanged between tasks and the more functions are involved. Especially when data has to be transmitted over an Ethernet-based communication network, the coordination between the processing tasks running on different end-devices and the communication network has to be ensured to meet strict end-to-end deadlines of task chains. In this work, we present a heuristic approach that computes schedules for distributed and data-dependent task chains consisting of preemptive and periodic tasks, taking into account the network communication delays of time-sensitive networks. Our algorithm is able to solve large problems for synthetic network topologies with randomized data dependencies in a few seconds. A high success rate was achieved, which can also be further enhanced by relaxing the deadline conditions.
\end{abstract}

\section{Index Terms-Cause-Effect-Chains, Real Time, Task Scheduling, Time-Sensitive Networks}

\section{INTRODUCTION}

$\mathbf{U}$ PCOMING innovations entail a steadily increasing amount of new functionalities, highly interconnected smart devices such as sensors and actuators, and an increasing volume of data transmitted over a communication network. In industrial automation, the future project Industry 4.0 is a known promoter of this trend. Industry 4.0 propels the digitization of industrial production and strives for greater connectivity between machines, actuators, sensors, and humans [1], [2]. In the automotive context, it is caused e.g. by the emergence of automated driving and advanced driver assistance systems. Both systems require a reliable perception of the environment with dozens of sensors like radar, LiDAR, and cameras [3], and a large processing effort. Also, the railway domain drives research to raise the grade of automation of the Automatic Train Operation (ATO) system to achieve a semi-automatic or even an unattended train operation [4]. To cope with the trends of the future, the communication network and the employed software platforms must be able to manage the increasing complexity. Especially when it comes to strict safety requirements, the temporal behavior of a system must still be reliable and deterministic.

With respect to the latest state in network communication systems, the Ethernet-based technology is established in a variety of domains with safety-related systems such as avionics, railway, industrial automation, and automotive. Ethernet itself provides stochastic access to the transmission media and is accordingly non-deterministic. The best-known Ethernet-based representatives that are capable to meet the requirements of safety-related real-time communication are the proprietary technologies Avionics Full-Duplex Switched Ethernet (AFDX), TTEthernet, and PROFINET. A common feature of these technologies is the provision of Quality

Anna Arestova, Wojciech Baron, Kai-Steffen Hielscher, and Reinhard German are with the University of Erlangen-Nürnberg, Erlangen 91058 Germany, (e-mail: \{anna.arestova, wojciech.baron, kai-steffen.hielscher, reinhard.german\}@fau.de) of Service (QoS) for specific traffic classes. A drawback of these technologies is vendor-dependency that complicates or makes the interoperability between devices of different manufacturers impossible. With the recent development within the IEEE 802.1 Working Group, Time-Sensitive Networking (TSN) [5] emerges as a promising candidate to implement the future communication systems for safety-critical domains, to establish a common and standardized development ground for future innovations, and to overcome the mentioned challenges. TSN provides mechanisms on top of Ethernet to guarantee reliable and real-time transmission of time-sensitive network traffic [6], [7]. At the same time, it enables the co-existence of network traffic with mixed criticality. Moreover, TSN in combination with Ethernet does not restrict the link speed, for example as opposed to PROFINET. It even supports link speeds greater or equal to 1 gigabit per second and thus provides high capacities for communication links. TSN is not only considered for industrial applications [8], it is also under investigation for automotive [9] and railway [10] use cases.

Nascent software platforms like the Automotive Open System Architecture (AUTOSAR) Adaptive platform especially advertise a flexible and simplified distribution of applications across the system and offer highly parallelized processing of data [11]-[13]. The goal is to be able to perform complex calculations like sensor fusion for automated driving or image processing in a short time. The trend is heading away from single-core and resource-constrained embedded systems that are mainly dedicated to a certain functionality. On the one hand, this development entails the major advantage of flexible task allocation to end-devices. On the other hand, due to the rising number of new functions and the enhanced use of multi-core systems, one processing task might have many competitors for computing and network resources and therefore the assignment of tasks to resources tends to become more challenging. The degree of complexity increases when task chains or Cause-Effect Chains (CECs) are involved. A CEC consists of a sequence of tasks that are bonded by data dependencies and that define a functionality together 
[14]. Consequently, proper coordination and scheduling is unavoidable for tasks organized in a CEC, particularly if the tasks have to meet temporal constraints.

Since CECs can have strict end-to-end timing requirements in real-time systems, the execution of the sequence of tasks that form a task chain must be well synchronized. It is especially important when considering the data age that covers the time interval from the generation of data by the first task to consumption by the last task in the chain. Tasks that are directly linked to each other by a precedence constraint can run on the same core, the same device, or different devices. Thus, if they are allocated to different devices, output data of one task has to travel across the communication network as a network stream or network task to be delivered to the next task in the task chain. The stream, in turn, might be exposed to conflict situations with other streams in the network. The scheduling or the analysis of the worst-case network delay of such a stream must be taken into account. Accordingly, the planning of CECs on computing and communication resources with a great conflict potential results in a complex mission and requires more detailed studies.

Mostly, combined task and network planning use exact methods such as Integer Linear Programming (ILP), which can lead to long runtimes. In this work, we address the combined planning of processing and network tasks and present a heuristic algorithm for scheduling CECs. The considered CECs consist of periodic processing and network tasks that are distributed in real-time systems with Ethernet networks combined with the IEEE 802.1Qbv TSN mechanism. We reference the proposed algorithm as ITANS (Incremental Task and Network Scheduling for Time-Sensitive Networks). The approach presumes that all execution times, static delays, precedence relationships within the task chains, and resource mappings are known beforehand. We will introduce an architectural and a common task model for processing and network tasks for distributed real-time systems. Moreover, we will demonstrate by means of selected characteristics from the automotive and railway domain that the ITANS heuristic allows to schedule more than a thousand processing and network tasks organized in CECs of different lengths and with different system configurations withing a few seconds.

The paper is organized as follows. In Section II] we introduce the applied TSN mechanisms. We present the system model in Section III and formulate the necessary scheduling constraints in Section IV. The heuristic approach is presented in Section V Moreover, we evaluate and reflect on the performance of ITANS in Section VI In Section VII we review the related work. We discuss and conclude our approach and give a preview of future activities in Section VIII.

\section{Fundamentals}

\section{A. Time-Sensitive Networking}

TSN enriches the IEEE 802.3 Ethernet with multiple latency control mechanisms. This allows to give better latency guarantees and, depending on the applied mechanism, also to promote low jitter to time-sensitive network traffic. Mechanisms such as frame preemption (IEEE 802.1 Qbu [15]), cyclic queuing

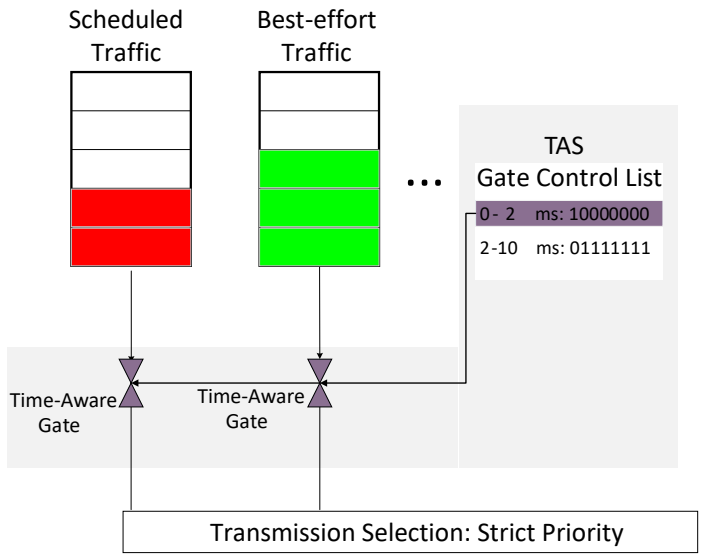

Fig. 1: TAS components, cf. [13].

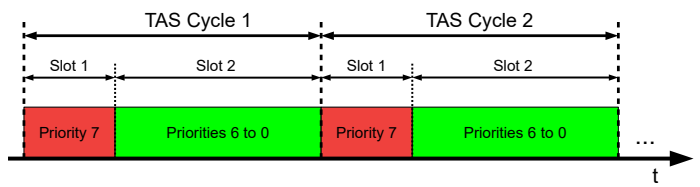

Fig. 2: Serialization on the wire, cf. [13].

and forwarding (IEEE 802.1 Qch [16]), asynchronous traffic shaping (IEEE 802.1 Qcr [17]), and Time-Aware Shaper (TAS) (IEEE 802.1 Qbv [18]) can facilitate these goals. In this work, we focus on the latter.

TAS introduces a Time Division Multiple Access (TDMA)based principle that enables the assignment of transmission slots to different traffic classes. Therefore, each egress queue of a physical network port that supports $802.1 \mathrm{Qbv}$ is preceded by a time-aware gate. The time-aware gates can open (state 1) or close (state 0) according to a cyclic schedule specified in the gate control list (GCL) (Fig. 1). Solely packets located in queues with open gates are considered for transmission by the transmission selection. If several queues are open, the transmission selection operates according to the established algorithm. In most cases, strict priority is applied.

An exemplary configuration of the TAS components is illustrated in Fig. 1 The critical traffic is transmitted in the relative interval $[0,2] \mathrm{ms}$ within a cycle of $10 \mathrm{~ms}$. The binary representation 10000000 shows the states of the time-aware gates. Each position represents one of 8 time-aware gates. In this example and also in our approach, the transmission selection policy is set to strict priority and one egress queue is dedicated for time- and safety-critical traffic. The resulting serialization on the wire is depicted in Fig. 2 The example from Fig. 1 shows a possible TAS configuration for one egress port. To be able to guarantee latency and jitter bounds for one or several streams, a feasible configuration of the gates associated with time-critical traffic has to be found on each egress port that is traversed by critical streams.

To avoid the intrusion of non-critical traffic into slots configured for critical traffic, guard bands are introduced. They prevent the transmission of packets a certain interval before the opening time of other gates. Such a safety interval may have the size of the transmission duration of a Maximum 
Transmission Unit (MTU). Further guard band mechanisms are described in [18]. TAS requires a common notion of time in all involved network devices and end-device. This can be ensured by the generalized Precision Time Protocol (PTP) specified in [19].

\section{B. TSN on the Data Link Layer}

TSN mechanisms are located on the data link layer in the Open Systems Interconnection (OSI) model. As TSN is part of the IEEE 802.1 Working Group, network streams that want to make use of TSN services carry a Virtual Local Area Network (VLAN) tag. This tag contains a priority code point (PCP) field that is used for traffic classification. The PCP value that has the integer range of 0-7 assigns a priority to the stream. Each priority value can be mapped to one egress queue if a physical port has eight egress queues, which usually applies. Additionally, TSN introduces the terms talker that refers to the source application and listener that identifies the destination application of a TSN stream. TSN streams can be propagated in a unicast or multicast fashion.

\section{TSN in end-devices}

TSN mechanisms not only apply to network devices but can also be configured in end-devices. Linux-based operating systems adopted special techniques to enable time-triggered injection of network traffic. The traffic control module tc of Linux allows to apply QoS mechanisms such as shaping, scheduling, policing, and packet dropping to network traffic [20]. The submodule qdisc (queuing discipline) allows the configuration of traffic control disciplines for each network interface. Queuing disciplines can control the behavior of the whole network interface card (NIC) or certain network classes. The queuing discipline of interest to achieve real-time behavior is Earliest TxTime First (ETF) [21]. ETF allows users to determine on application level the time when a network packet of a certain traffic class should be dequeued from the traffic control buffer into the hardware queue of a NIC. Furthermore, it gives users the ability to specify the time when the packet should leave the NIC if the function offload is specified and supported [21]. Special network drivers are necessary to realize the ETF functionality. Additionally, the system clock and the hardware clock of the NIC have to be synchronized in order to achieve the desired behavior.

The mapping of socket priorities to traffic classes and mapping of traffic classes to hardware queues can be done either by the Multiqueue Priority (MQPRIO) queuing discipline [22] or the Time Aware Priority Shaper (TAPRIO) queuing discipline [23]. Both disciplines are classfull, i.e. they are able to create classes to which other queuing disciplines can be applied. ETF, in contrast, works on a traffic class. TAPRIO additionally provides an implementation of the TAS operating principle as a software solution (at this point in time). When using TAPRIO, it is possible to configure time slices for network classes of an egress port. These time slices determine the interval when packets of a certain class are forwarded to the NIC.

Queuing disciplines can be combined by switching them hierarchically. When TAPRIO acts as the superior discipline and
ETF is applied to one or multiple classes of TAPRIO, TAPRIO controls the transmission windows of different traffic classes and ETF specifies when a certain packet of a traffic class is forwarded to the NIC and put on the wire [24], [25]. The combination of MQPRIO and ETF allows the classification of outgoing network traffic and the timely injection of individual packets of a certain traffic class on the wire. MQPRIO does not subdivide the time space into transmission windows for dedicated traffic classes. We refer at least to the MQPRIO-ETF combination in our scheduling approach. However, we prefer and advise using the TAPRIO-ETF configuration to avoid the collision with packets from non-critical traffic classes that are generated in the same end-device. Our goal is to specify the transmission offset and deterministic travel time through the communication network for each TSN stream with the ITANS algorithm.

\section{SYSTEM MODEL}

First, we introduce a system model for a distributed realtime system consisting of processing and network resources and tasks. We divide the system model into an architectural model and a task model. We adopt the concept of a common representation of processing and network tasks from [26].

\section{A. Architectural Model}

We denote the architectural view of the system as a graph $\mathcal{G}(\mathcal{V}, \mathcal{L})$ consisting of vertices $\mathcal{V}$ and links $\mathcal{L}$. The set $\mathcal{V}$ comprises end-devices, denoted as $v_{i}^{e} \in \mathcal{V}$, and network switches, represented by $v_{i}^{s} \in \mathcal{V}$. Each end-device node $v_{i}^{e}$ owns one or several processing cores. The devices are physically interconnected by bi-directional full-duplex Ethernet links. For each physical link between two distinct nodes $v_{j}$ and $v_{k}(j !=k)$ the set $\mathcal{L}$ contains two directed logical Ethernet links $l_{j, k}$ and $l_{k, j}$. The links uniquely identify the physical source and destination ports. Each logical link $l_{j, k}$ is characterized by the tuple $\left\langle r_{j, k}, s_{j, k}\right\rangle$, with $r_{j, k}$ describing the link's data rate in bits per second and $s_{j, k}$ denoting the length of the link in meter.

\section{B. Task Model}

The task set $\mathcal{T}$ is divided into the representation of processing tasks $\tau_{i}^{p} \in \mathcal{T}$, and network tasks $\tau_{i}^{n} \in \mathcal{T}$. Each processing task $\tau_{i}^{p}$ is defined by the tuple $\left\langle p_{i}, \phi_{i}, d_{i}, e_{i}^{j}, \omega_{i}^{j}\right\rangle$, with $p_{i}$ denoting the task period, $\phi_{i}$ the release offset referred to the relative beginning $t^{\prime}=0$ of $p_{i}$, and $d_{i}$ the relative deadline. The worst-case execution time (WCET) is depicted by $e_{i}^{j}$ and the worst-case response time (WCRT) is denoted by $\omega_{i}^{j}$. Index $j$ refers to the end-device $v_{j}^{e}$ and expresses that WCET and WCRT are hardware-dependent. The WCRT comprises the difference between the release time $\phi_{i}$ and the completion time of the task that is $\phi_{i}+e_{i}^{j}$ plus the delay $D_{\text {preempt }}^{i}$ due to preemption by other tasks and the scheduling overhead $D_{\text {sched }}$. The preemption delay depends on the number of tasks on the same core that have a higher priority and whose execution overlap with the task of interest. The scheduling overhead arises due to context switches and depends on the hardware and the operating system (OS). 


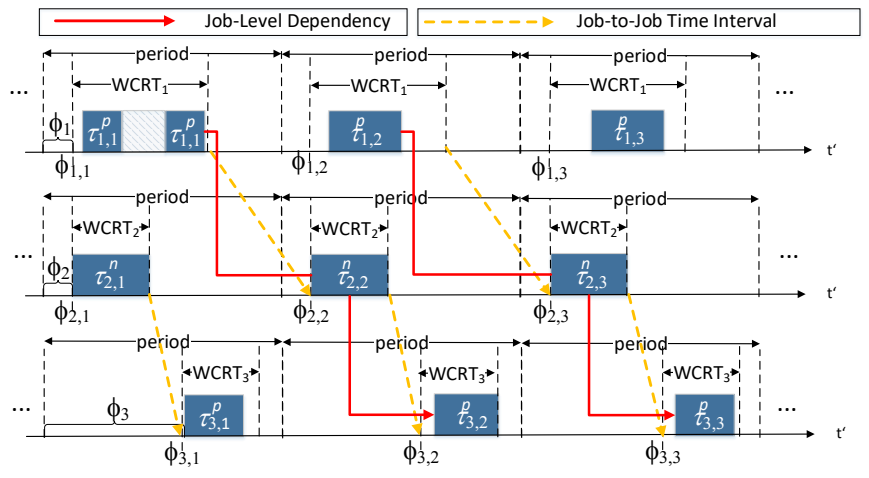

Fig. 3: Job-level dependencies for $\tau_{1} \prec \tau_{2} \prec \tau_{3}$.

Whenever tasks are connected by a precedence order due to data dependencies, they form a CEC [27]. It means that tasks supply data to their immediate successor tasks. Additionally, we introduce the term job that describes a task in execution. $\tau_{i, j}$ is the $j$-th execution or job of task $\tau_{i}$. Thus, $\phi_{i}$ can be adjusted to $\phi_{i, j}$ of the $j$-th instance, which corresponds to $\phi_{i, j}=\phi_{i}+(j-1) \times p_{i}$ since the first release $(j=1)$ of task $\tau_{i}$. Other task characteristics remain unchanged in all executions. We refer to the dependencies between jobs of the tasks of one CEC as job-level dependencies [27]. Since we presume that all tasks in a CEC have the same period, the relative time intervals between jobs organized in a job-level dependency are the same for all job-level dependencies. The job-level dependency consisting of the jobs $\left\{\tau_{1,1}^{p}, \tau_{2,2}^{n}, \tau_{3,2}^{p}\right\}$ in Fig. 3 has the same temporal distances between the jobs as the job-level dependency $\left\{\tau_{1,2}^{p}, \tau_{2,3}^{n}, \tau_{3,3}^{p}\right\}$.

One CEC cec $_{i} \in \zeta$ is captured by the tuple $\left\langle T_{i}, e 2 e_{i}\right\rangle$, with $T_{i}$ comprising all processing and network tasks that are related by data dependencies and $e 2 e_{i}$ denoting the maximum allowed end-to-end duration from the release time of the first job in a job-level dependency until the completion of the last job or jobs in a job-level dependency. If any processing task of a CEC is located on a different end-device than its preceding task, data has to be delivered over a communication network by a network task. As illustrated in Fig. 4 task $\tau_{1}^{p}$ has two successor tasks $\tau_{2}^{p}$ and $\tau_{3}^{p}$. While $\tau_{1}^{p}$ and $\tau_{2}^{p}$ share the same end-device $v_{1}^{e}, \tau_{3}^{p}$ has been allocated to end-device $v_{2}^{e}$. Thus, the network task $\tau_{4}^{n}$ has to be inserted and becomes the immediate successor of $\tau_{1}^{p}$ and the new predecessor of $\tau_{3}^{p}$. The identification of the predecessors (previous tasks) of an allocated task is determined by the function prev $: \mathcal{T} \longrightarrow \mathcal{T}$. The determination of successors (next tasks) of a task is defined by the mapping next $: \mathcal{T} \longrightarrow \mathcal{T}$.

The network task set comprises TSN streams that arise due to allocation of CEC tasks to different nodes. We assume that each TSN stream consists of one Ethernet frame to simplify the characterization. To unify the representation of processing and network tasks, each stream or network task $\tau_{i}^{n}$ is characterized by the tuple $\left\langle p_{i}, \phi_{i}, d_{i}, e_{i}^{j}, \omega_{i}^{j}, s_{i}, p t_{j}\right\rangle$, with $p_{i}$ denoting the period, $\phi_{i}$ the relative transmission start offset referred to the beginning of $p_{i}$, and $d_{i}$ the maximum allowed end-to-end network latency. In contrast to processing tasks, $e_{i}^{j}$ determines the worst-case transmission time (WCTT) through

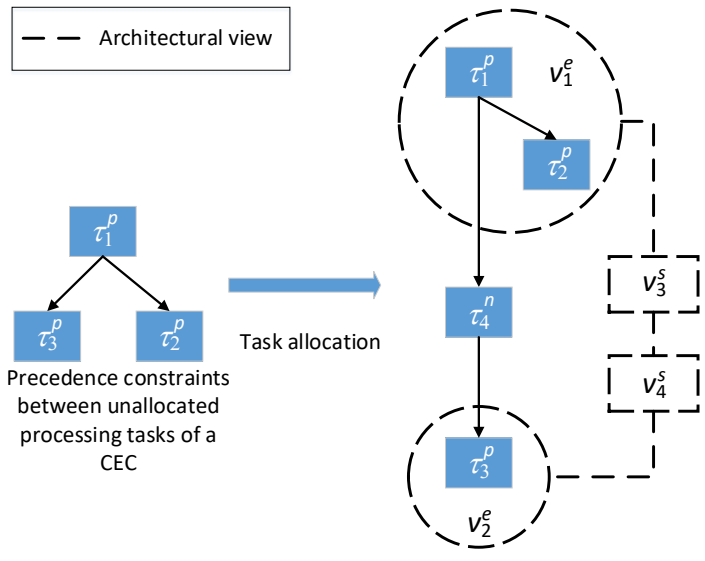

Fig. 4: CEC allocation to end-devices

the communication network to the sink $v_{j}^{e}$ starting from the source node $v_{k}^{e}$. We pursue a stream isolation principle introduced in [28] and discussed in our former work [29] where streams get exclusive access to all communication links of their path as soon as they are injected onto the wire by the talker node. It means that they will not experience any interference with other streams and they do not have to wait in egress queues of the traversed ports. Thus, preemption of network tasks is not allowed and consequently the WCRT $\omega_{i}^{j}$ of $\tau_{i}^{n}$ equals the WCTT $e_{i}^{j}$. We maintain the index $j$ of the node for the WCTT and WCRT to clarify that if the stream has multiple listeners (consuming applications) $e_{i}^{j}$ and $\omega_{i}^{j}$ may differ for each listener if the listeners are located on different nodes. The size of the stream in Byte is defined by $s_{i}$ and $p t_{j}$ specifies the stream path. $p t_{j}$ consists of an ordered sequence of Ethernet links included in $\mathcal{L}$ starting from the talker node $v_{k}^{e}$ and reaching to the listener node $v_{j}^{e}$. It is included in the list $\mathcal{P}_{i}$ that contains the paths to all listeners. Furthermore, we introduce a mapping of the stream to the talker node and the listener nodes. The assignment of a talker node to the stream is captured by the function talkV: $\mathcal{T} \longrightarrow \mathcal{V}$. The mapping of listener nodes to a stream is defined by listsV $: \mathcal{T} \longrightarrow \mathcal{V}$. The talker application of $\tau_{i}^{n}$ can be retrieved by $\operatorname{prev}\left(\tau_{i}^{n}\right)$ and the listeners by $\operatorname{next}\left(\tau_{i}^{n}\right)$ accordingly.

Fig. 5 shows some characteristics of the network task $\tau_{2}^{n}$. The talker and at the same time the predecessor task of $\tau_{2}^{n}$ is $\tau_{1}^{p}$. The listeners are represented by the processing tasks $\tau_{3}^{p}$ and $\tau_{4}^{p}$. The listener nodes $v_{3}^{e}$ and $v_{4}^{e}$ can be retrieved with the function $\operatorname{lists} V\left(\tau_{2}^{n}\right)$. Each listener has its individual WCTT and WCRT. The path $p t_{3}$ from $v_{1}^{e}$ to $v_{3}^{e}$ is described by the ordered sequence $\left\{l_{1,2}, l_{2,3}\right\}$ and the path $p t_{4}$ from $v_{1}^{e}$ to $v_{4}^{e}$ is described with $\left\{l_{1,2}, l_{2,4}\right\}$.

\section{FORMAL CONSTRAINTS}

We declare a resulting ITANS schedule as feasible if the following set of constraints is fulfilled. The constraints describe the required timing behavior of the set of tasks to be scheduled. We divide them into CEC and task constraints. 


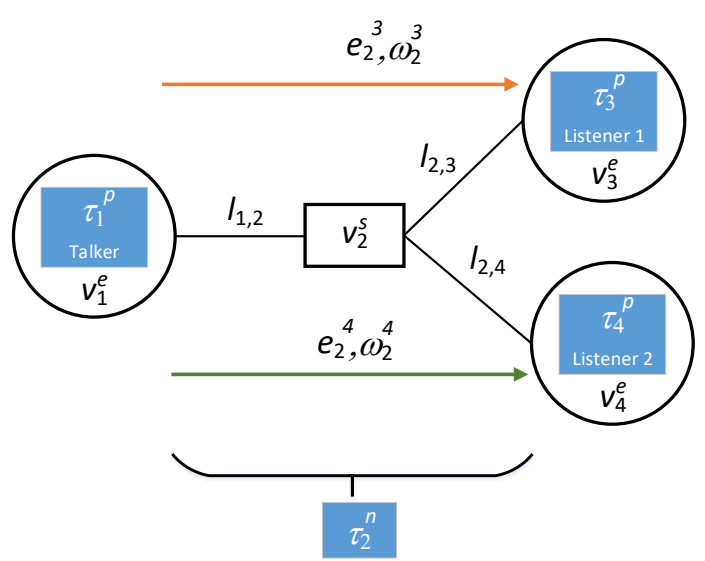

Fig. 5: Example of network task characteristics.

\section{A. CEC Constraints}

We distinguish between processing and network tasks. Each CEC has one producer task and a variable number of intermediate and last tasks. The producer task and all last tasks of a task chain are always processing tasks. Intermediate tasks can be processing or network tasks. Each network task has exactly one predecessor task and a variable number of successor tasks. The predecessor and the successors of a network task are processing tasks. A processing task can follow and precede a variable number of processing tasks (same end-device) as well as network tasks. Moreover, all tasks of a CEC are constrained to have the same (homogeneous) period. The periods of different CECs are harmonic, i.e. they are integer multiples of each other. All CECs are loop-free.

For each CEC $\operatorname{cec}_{i}$ we have to determine the jobs of the tasks $\in T_{i}$ that form a job-level dependency and minimize the end-to-end latency as illustrated in Fig. 3 and Fig. 6 . Due to long task chains, i.e. several chained tasks that are related by precedence constraints, not all jobs that are part of a job-level dependency can be executed within the same cycle. Still, we aim to schedule subsequent jobs of a job-level dependency within the same cycle whenever it is possible to minimize the end-to-end time $e 2 e_{i}$. Otherwise, the execution of succeeding jobs takes place in the subsequent cycle or the one after that if the execution of a job starts in one cycle and finishes in the next cycle. Fig. 6 depicts the latter scenario where job $\tau_{1, k}^{p}$ starts in cycle $k$ and finishes in cycle $k+1$. Consequently, the first and the last jobs in the same job-level dependency may reveal an even greater cycle offset. We introduce the variable $\gamma_{k}$ that indicates in which cycle a job of task $\tau_{k}^{p}$ is released referred to the release cycle of the job of the first task $\tau_{a}^{p}$ in the same job-level dependency. The cycle offset $\gamma_{a}$ of any job of the leading task $\tau_{a}^{p}$ is always 1 regardless of the absolute time. In Fig. 6, job $\tau_{1, k}^{p}$ is the first job in a job-level dependency and thus $\gamma_{1}$ equals 1 . Job $\tau_{2, k+2}^{p}$ receives data from $\tau_{1, k}^{p}$. Cycle offset $\gamma_{2}$ corresponds to 3 because $\tau_{2, k+2}^{p}$ is released two cycles later than the preceding job $\tau_{1, k}^{p}$ in the same job-level dependency.

Since the cycle offset $\gamma$, the relative offset $\phi$, and the WCRT $\omega$ are identical in each execution cycle for all tasks, we do not have to consider all job-level dependencies individually.

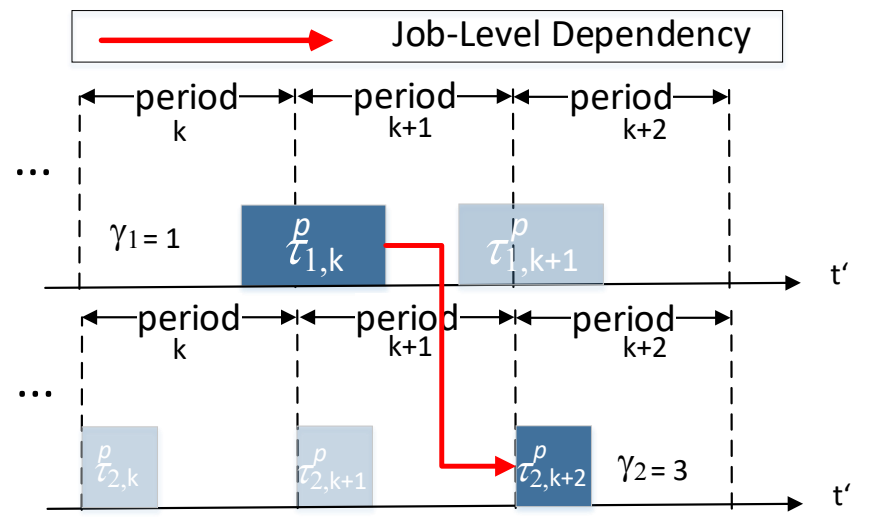

Fig. 6: Job-level dependency between $\tau_{1}$ and $\tau_{2}$.

Without limiting the generality, we will impose the following constraints only for the job-level dependency that starts with the first job $\tau_{a, 1}$ of the first task $\tau_{a}$ of a CEC.

In Eq. (1) we match the jobs of the first task and its direct successors that build a job-level dependency. Therefore, we formulate the precedence constraint for the immediate successors, each denoted as $\tau_{k}$, of the first task $\tau_{j}^{p}$ of each CEC cec $_{i}$ and determine $\gamma_{k}$ for each successor task:

$$
\begin{aligned}
& \forall c e c_{i} \in \zeta, \forall \tau_{k} \in \operatorname{next}\left(\tau_{a}^{p}\right), \tau_{a}^{p}=\operatorname{first}\left\{T_{i}\right\}, \gamma_{a}=1, \\
& \gamma_{k} \geq \gamma_{a}: \underset{\gamma_{k}}{\arg \min }\left\{\phi_{k, \gamma_{k}}: \phi_{k, \gamma_{k}} \geq \phi_{a, \gamma_{a}}+\omega_{a}^{j}+D_{c o m m}\right\}
\end{aligned}
$$

where any successor $\tau_{k, \gamma_{k}}$ should not be released until the predecessor job $\tau_{a, \gamma_{a}}^{p}$ has finished its execution considering the WCRT $\omega_{a}^{j}$ of $\tau_{a}^{p}$ and the additional delay $D_{\text {comm }}$. The function first $\left\{T_{i}\right\}$ returns the first task of the set of tasks $T_{i}$ of $c e c_{i}$. The index $j$ in $\omega_{a}^{j}$ indicates that $\tau_{a}^{p}$ is running on device $v_{j}^{e}$. We maintain the index $j$ to have a common expression for network and processing tasks. Network tasks depend on the index as it indicates the destination node of one of its listeners and the WCRT might differ for each listener. The delay $D_{\text {comm }}$ describes the delay caused by the inter-process communication if $\tau_{a}^{p}$ and $\tau_{k}$ are both processing tasks. Otherwise, $D_{\text {comm }}$ represents the delay caused by the communication stack when packing or unpacking a network packet and the delay caused by the NIC before sending or receiving the packet. If the predecessor is a processing task and the successor a network task then $D_{\text {comm }}$ depends on the device that the processing task is running on. Vice versa $D_{\text {comm }}$ depends on the listener's node of the network task.

Eq. (2) describes how a job-level dependency and a precedence relationship are defined when $\tau_{k}$ is an intermediate or the last task of $\operatorname{cec}_{i}$ and $\tau_{m}$ is one of its predecessors. The variable $\gamma_{k}$ depicts the cycle offset of $\tau_{k}$ and $\gamma_{m}$ denotes the cycle offset of $\tau_{m}$. To satisfy the precedence constraint, job $\tau_{k, \gamma_{k}}$ cannot start until all of its predecessors have finished including the WCRT of the predecessors and the additional delay $D_{\text {comm }}$. The cycle offsets of $\tau_{k}$ and of its predecessors can differ but have to be assumed as small as possible to build 
a valid job-level dependency. In this case, $\omega_{m}^{j}$ represents the WCRT of a processing or network task.

$$
\begin{aligned}
& \forall \text { cec }_{i} \in \zeta, \tau_{k} \in T_{i}, \operatorname{prev}\left(\tau_{k}\right) \neq \emptyset, \gamma_{k} \geq \gamma_{m}: \\
& \max _{\text {prev }}=\max _{\tau_{m} \in \operatorname{prev}\left(\tau_{k}\right)}\left(\phi_{m, \gamma_{m}}+\omega_{m}^{j}\right)+D_{\text {comm }} \\
& \underset{\gamma_{k}}{\arg \min }\left\{\phi_{k, \gamma_{k}}: \phi_{k, \gamma_{k}} \geq \max _{\text {prev }}\right\} .
\end{aligned}
$$

Each CEC has an end-to-end deadline constraint on the job-level dependency. The difference between the release time $\phi_{a, \gamma_{a}}$ of the first job and the end time of each last job $\tau_{k, \gamma_{k}}^{p}$ in the same job-level dependency has to be less than or equal to the specified end-to-end latency $e 2 e_{i}$ :

$$
\begin{aligned}
& \forall \operatorname{cec}_{i} \in \zeta, \tau_{a}^{p}=\operatorname{first}\left\{T_{i}\right\}, \gamma_{a}=1, \gamma_{k} \geq \gamma_{a}: \\
& \max _{\tau_{k}^{p} \in \operatorname{last}\left(T_{i}\right)}\left(\phi_{k, \gamma_{k}}+\omega_{k}^{j}\right)-\phi_{a, \gamma_{a}} \leq e 2 e_{i} .
\end{aligned}
$$

The function $\operatorname{last}\left(T_{i}\right)$ returns the tasks without successors within the task set $T_{i}$ of $\mathrm{cec}_{i}$.

\section{B. Task Constraints}

Each processing and network task has to be executed once in its period. Processing tasks are allowed to start in one period and to finish in the consecutive one, as shown in Fig. 6. Thus, if the task $\tau_{i}$ has a relative offset $\phi_{i}>0$, we adjust $d_{i}$ that is originally equal to $p_{i}$ to $d_{i}=p_{i}+\phi_{i}$. Network tasks are restricted by the common network cycle TASCycle that is discussed in Section V-D. Thus, $d_{i}$ of a network task $\tau_{i}^{n}$ is $d_{i}=T A S C y c l e$. The period constraint is described with:

$$
\forall \tau_{i} \in \mathcal{T}: \phi_{i}+\omega_{i}^{j} \leq d_{i} .
$$

Eq. (4) applies to all task executions. Processing tasks on the same processing core can preempt each other. Network tasks are planned collision-free according to the stream isolation principle. Thus, the dynamic queuing delay $D_{q}^{l_{x, y}}$ on the link $l_{x, y}$ assumes zero. This measure contributes to less jitter and more determinism, as the remaining delays in the network are static. But it also requires a careful planning approach. As already described in our previous work [30], the static network delays cover the transmission delay $D_{\text {tans }, i}^{i_{x, y}}=\left(s_{i} \times 8 b i t / B y t e\right) / r_{x, y}$ of network task $\tau_{i}^{n}$ on link $l_{x, y}$, the propagation delay $D_{\text {prop }}^{l_{x, y}}$ in dependence of the cable length $s_{x, y}$ and material composition, and the processing delay $D_{\text {proc }}^{x}$ on a network node $v_{x}^{s}$. $D_{\text {proc }}^{x}$ depends on the switching factory. The WCRT $\omega_{i}^{j}$ of a network task $\tau_{i}^{n}$ from the talker to a listener is formed as follows:

$$
\begin{aligned}
& \forall v_{j}^{e} \in \operatorname{listsV}\left(\tau_{i}^{n}\right), p t_{j} \in \mathcal{P}_{i}: \\
& \text { sum }_{\text {links }}=\sum_{l_{x, y} \in p t_{j} \backslash\left\{\operatorname{first}_{\left.\left(p t_{j}\right)\right\}}\right.} D_{\text {proc }}^{x}+D_{\text {trans }, i}^{l_{x, y}}+D_{\text {prop }}^{l_{x, y}} \\
& \omega_{i}^{j}=D_{\text {trans }, i}^{\text {first }\left(p t_{j}\right)}+D_{\text {prop }}^{\text {first }\left(p t_{j}\right)}+\text { sum }_{\text {links }},
\end{aligned}
$$

with $v_{j}^{e}$ specifying a listener's node and $p t_{j}$ denoting the stream path of the network task $\tau_{i}^{n}$ to the listener's node $v_{j}^{e}$.

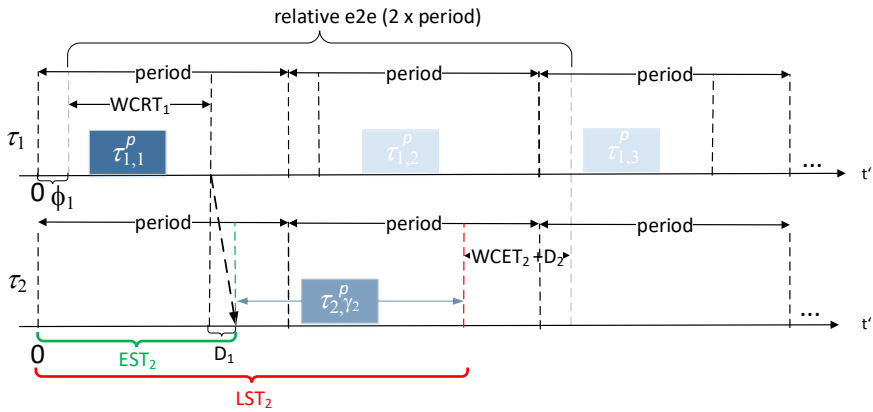

Fig. 7: Determination of $E S T_{2}$ and $L S T_{2}$ for $\tau_{1} \prec \tau_{2}$

The function $\operatorname{first}_{(}\left(p t_{j}\right)$ returns the first Ethernet link of the set $p t_{j}$ consisting of ordered links. Eq. (5) indirectly includes the constraint, that the transmission on an intermediate or last link $l_{x, y}$ cannot start until all bits of the stream have been pushed on the previous link, the stream has been propagated over the previous link, and before it has been processed on the source node $v_{x}^{s}$ of the regarded link $l_{x, y}$. It is also evident from the formulas that we focus on store-and-forward switches.

\section{Scheduling Algorithm}

The proposed scheduling algorithm includes both the planning of processing tasks and the scheduling of network tasks on their assigned resources. While processing tasks allow preemption, network tasks have to run without interference. The goal of the algorithm is to schedule the tasks of all CECs so that the aforementioned constraints are met. We do not provide solutions for the route planning of streams, nor do we optimize the allocation of tasks to resources. However, we presume a proper allocation of tasks to resources and fixed stream paths.

\section{A. Earliest and Latest Start Time}

For each task that has at least one predecessor task, we determine the earliest start time (EST) and the latest start time $(L S T)$. These parameters define the scope and the mobility for the scheduling of a task. $E S T_{i}$ of task $\tau_{i}$ is constrained by the completion time of its predecessors. Thus, the predecessors have to be planned first and $E S T_{i}$ is computed during the scheduling process. $E S T_{i}$ and $L S T_{i}$ relate to the relative time $t^{\prime}=0$, as depicted in Fig. 7. In absolute time, $t^{\prime}=0$ determines the beginning of the release cycle of the producer job $\tau_{a, 1}$ that is an integer multiple of $p_{a}$. This leads to the following formula for $E S T_{i}$ of task $\tau_{i}$ if the task has at least one predecessor $\tau_{k}$ :

$$
\begin{aligned}
& \forall \tau_{i} \in \mathcal{T}, \operatorname{prev}\left(\tau_{i}\right) \neq \emptyset, \gamma_{k} \geq 1: \\
& E S T_{i}=\max _{\tau_{k} \in \operatorname{prev}\left(\tau_{i}\right)} \phi_{k, \gamma_{k}}+\omega_{k}^{j}+D_{\text {comm }}
\end{aligned}
$$

where $\gamma_{k}$ denotes the cycle offset of $\tau_{k}$ and $\omega_{k}^{j}$ the WCRT of $\tau_{k}$. The $L S T_{i}$ represents the latest start time of $\tau_{i}$ relative to $t^{\prime}=0$ within the job-level dependency. It can be calculated solely with the knowledge of the maximum end-to-end delay of the CEC and the precedence relationships in a job-level 
dependency before the actual scheduling process. The $L S T$ of the last task is the relative end-to-end time minus the WCET or WCTT and the overhead delay caused by the hardware or the OS. The $L S T$ of an intermediate task is derived from the $L S T$ of the successor with the smallest $L S T$ value:

$$
\begin{aligned}
& \forall \tau_{i} \in \mathcal{T}, \operatorname{prev}\left(\tau_{i}\right) \neq \emptyset, \operatorname{next}\left(\tau_{i}\right) \neq \emptyset: \\
& L S T_{i}=\min _{\tau_{k} \in \operatorname{next}\left(\tau_{i}\right)} L S T_{k}-\left(e_{i}+D_{i}\right),
\end{aligned}
$$

where $\tau_{k}$ is a successor task of $\tau_{i}$. $D_{i}$ summarizes the occurring overhead delays arisen due to inter-process communication, the communication stack, and scheduling. Since the calculation takes place before the actual scheduling process, we do not yet know the WCRT of the tasks. If the first task $\tau_{a}^{p}$ in the task chain experiences an offset $\phi_{a}>0$ during the task planning process, then the relative end-to-end time and the $L S T$ values of all immediate and indirect successors will be aligned by $\phi_{a}$ subsequently. As illustrated in Fig. 7, the $L S T_{2}$ of $\tau_{2}^{p}$ is determined by $e 2 e+\phi_{1}-\left(W C E T_{2}+D_{2}\right)$. The successor job $\tau_{2, \gamma_{2}}^{p}$ of $\tau_{1,1}^{p}$ can start its execution in the first cycle or the second, as defined by the scope of $E S T_{2}$ and $L S T_{2}$. Consequently, $\gamma_{2}$ equals 1 or $2 . E S T_{2}$ results from the finishing time of $\tau_{1,1}^{p}$ and the overhead delay $D_{1}$.

The difference between the LST and EST defines the mobility of a task. The tighter the end-to-end constraint of a CEC, the smaller the difference between $E S T$ and $L S T$. If $L S T_{i}-E S T_{i} \geq p_{i}$, then $\phi_{i}$ of task $\tau_{i}$ can take any value in the range $\left[0, p_{i}\right.$. Otherwise, $\phi_{i}$ has to take one of the following values: $\phi_{i} \in\left[E S T_{i}, L S T_{i}\right] \% p_{i}$. This value has to be adjusted to the timer resolution of the end-device. Each producer task $\tau_{a}$ is initialized with $E S T_{a}=0$ and $L S T_{a}=p_{a}$. The resulting start time of the job $\phi_{k, \gamma_{k}}$ from the scheduling process lies between $E S T_{k}$ and $L S T_{k}$.

\section{B. Allocation of Time Resources}

We manage all processing and network resources in time slots. The time slots can be occupied or free. Each time slot is described by the size in microseconds. Each occupied slot is associated with a task. We refer to the smallest organizing unit of processing and network task resources as a management unit. Each management unit organizes its free and occupied slots. Computational cores are the resources of the processing tasks and communication links are referred to as the resources of network tasks. One management unit on a computational core has the size of the smallest period among all tasks running on this core. On an egress port, that is the source of a link, one management unit has the size of the smallest occurring task period TASCycle in the network.

A single processing task can occupy one slot or several split slots per period if preemption is allowed. A network task can only have one slot per period and the slots have to be equidistant within the hyperperiod of the network that is the least common multiple of all network task periods. This measure serves to comply to the stream isolation principle and to avoid dynamic queuing delays.

The following procedures are explained in advance to better understand the overall algorithm from Section V-F

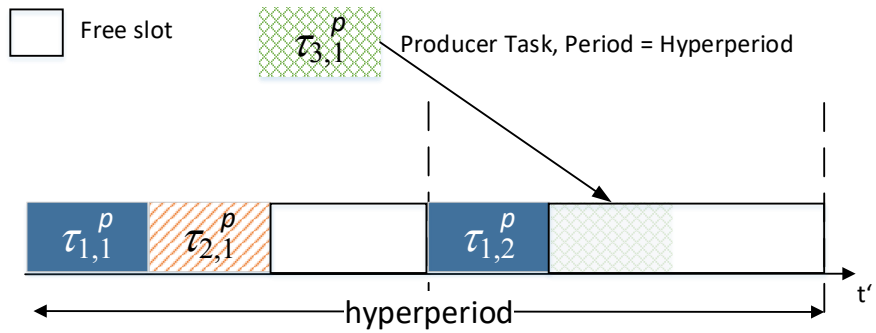

Fig. 8: Distribution of producer tasks.

\section{Management of Processing Task Resources}

The slotSearchProc method applied in Section V-F schedules one task $\tau_{i}^{p}$ on its computing resource $c_{i}$. The function looks for fitting free slots for task $\tau_{i}^{p}$ in the interval of one period $p_{i}$ and within the hyperperiod $H P$ of all task periods that are executed on the same core $c_{i}$. Hence, $\tau_{i}^{p}$ has to be scheduled $H P / p_{i}$ times per hyperperiod. We distribute the producer tasks that have a higher cycle time than the size of one management unit on $c_{i}$ such that the occupancy of the management units is similarly balanced across the hyperperiod. The example in Fig. 8 shows that the producer task $\tau_{3}^{p}$ has a period that is equal to the hyperperiod. The smallest period in this scenario is $H P / 2$ defined by task $\tau_{1}^{p} . \tau_{3}^{p}$ is scheduled in the second management unit. This has the advantage that tasks that are scheduled later and are restricted by $E S T$ and $L S T$ values can potentially find free slots more easily, especially if they have a small period compared to the hyperperiod. Intermediate and last tasks in a task chain begin to look for slots at the management unit indexed with $\lfloor(E S T \% H P) /$ size of management unit $\rfloor+1$. The indexing starts with the value 1 . The initial search offset is EST\%size of management unit. The search offset adapts throughout the process. From there on, further slots are searched at intervals of the task period. The EST value has to be translated to the hyperperiod because long task chains can exceed the hyperperiod. In Fig. 11, the algorithm would start the slot search for task $\tau_{3}^{p}$ at the management unit that results from $E S T_{3} . E S T_{3}$ is located at the end of job $\tau_{2,2}^{n}$ plus overhead delay. After finding slots that fit the $E S T_{3}$ and $L S T_{3}$ bounds, further slots have to be found throughout the hyperperiod. If the first regarded management unit has the highest index among all management units the algorithm will go back to the management units at the first index, then at the second, etc., until enough resources have been found. The execution of task can take place across slots of different management units. At any time the period constraint from Eq. (4) must be ensured. This means, that the slots are not allowed to overlap.

Whenever a task finds fitting slots within the hyperperiod, the affected free slots are reduced by the size of the task (WCET) and $D_{\text {sched }}$ of the system. This leads to new occupied slots that refer to the planned task. On success, the slotSearchProc function sets the earliest release time $\phi_{i}$ and the maximum WCRT $\omega_{i}^{j}$ on device $v_{j}^{e}$ within the hyperperiod, as well as the cycle offset $\gamma_{i}$ of the task to specify the joblevel instance. Each planned task is assigned a priority that is 


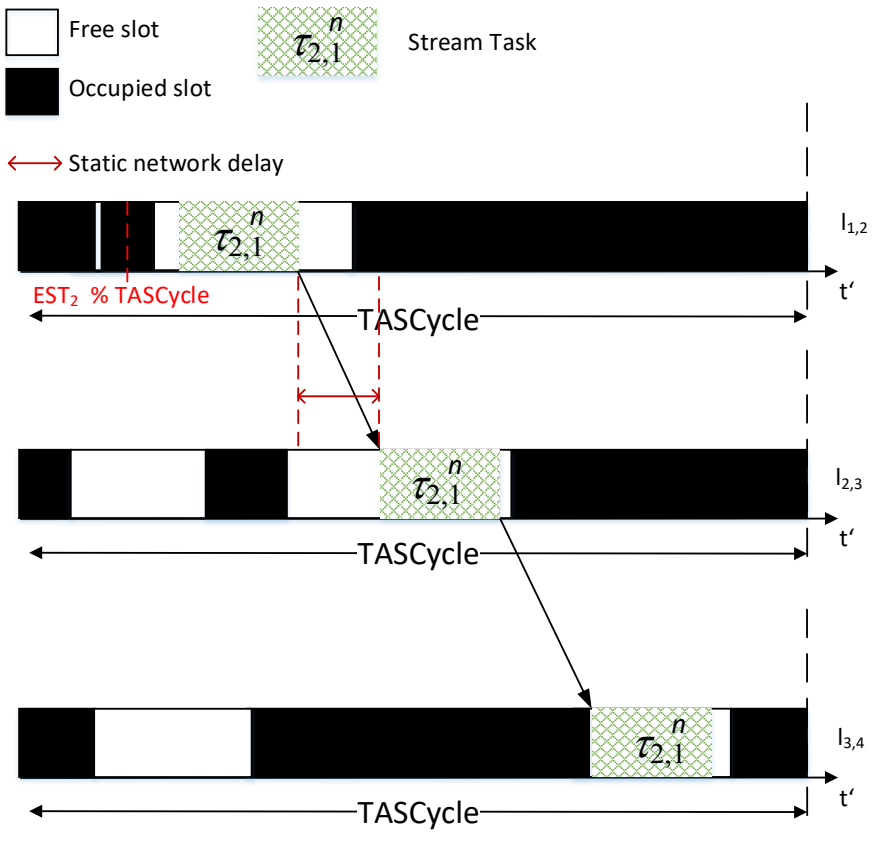

Fig. 9: Example of slot search for a network task.

the consecutive number in planning order, which is managed for each core independently. $\tau_{i}^{p}$ from Fig. 8 gets the priority 3 for instance. The priority is higher the smaller the value is. If slots that cover the task's size and overhead are not available, the algorithm looks for a composition of free slots comprising the duration of the task and each considering the overhead delays. Composite slots indicate task preemption. Whether the preemption occurs depends on the actual execution time that is less than or equal to the WCET.

\section{Management of Network Task Resources}

The stream planning is more complex as one stream has to be planned across several links. Thus, on each link free slots have to be detected that occupy the size of the stream. The size comprises the transmission duration of the stream on the link plus the interframe gap in our case. Physically, we look for resources on the source port of the link. The slots on subsequent links must be properly aligned. The task of investigation can be aligned within the free slots. Fig. 9 shows a scheduling example of network task $\tau_{2}^{n}$ on the path $\left\{l_{1,2}, l_{2,3}, l_{3,4}\right\}$. If there is a fitting slot on $l_{1,2}$, it means that the combination of slots on subsequent links is valid if the slot on the adjacent link $l_{2,3}$ starts exactly at the same time or before the stream arrives at the egress queue of the source port of $l_{2,3}$. The arrival time on $l_{2,3}$ includes the static network delay that comprises the transmission and propagation on $l_{1,2}$ and the processing on device $v_{2}^{s}$ (source node of $l_{2,3}$ ). The same rule applies to the links $l_{2,3}$ and $l_{3,4}$. The occupied slots are representatives of GCL entries. After finding fitting slots, the GCL entries on the corresponding devices will be configured such that the gates for the critical egress queues will be opened when the slot starts (see Section V-E). If the stream arrived before the start of a slot, it would have to wait. This situation would result in the need to buffer the stream and in a queuing

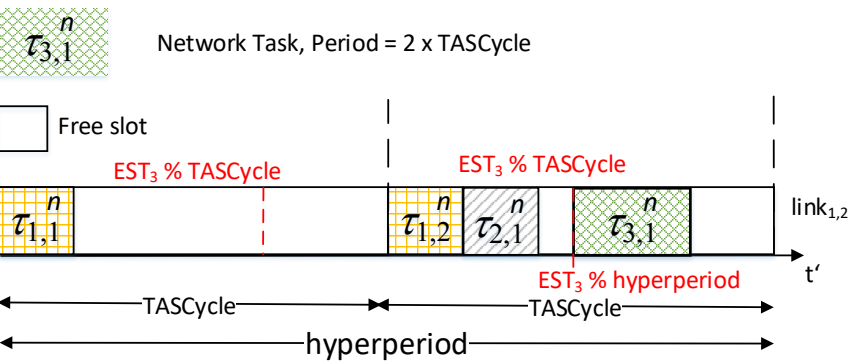

Fig. 10: Example of stream planning.

delay. Consequently, this would violate the stream isolation principle. Extending ITANS to consider queuing delays is a subject of future work.

For the whole network, we determine a common scheduling cycle $T A S C y c l e$ that corresponds to the smallest period of all occurring critical streams/network tasks in the network. The periods of critical streams must be a multiple of TASCycle. We refer to the harmonic periods which are also a subject in PROFINET [31] or TTEthernet [32]. Non-harmonic periods contribute to more complexity and make it more difficult to find a valid schedule. Similar to the management of processing task resources, we handle the free and occupied slots over the hyperperiod of the network. Let us assume that the network cycle is $1 \mathrm{~ms}$ and the hyperperiod is $4 \mathrm{~ms}$. A network task with a period of $1 \mathrm{~ms}$ has to find equidistant free slots over the hyperperiod, thus in all 4 management units and on all links. A network task with a period of $4 \mathrm{~ms}$ has to be scheduled in one of four management units on each link. The restriction defined in Eq. (4) must be taken into account. It ultimately depends on the temporal requirements, in our case the EST and $L S T$, which management units can be considered for scheduling.

Let us consider the following example in Fig. 10. $p_{3}=$ $2 \mathrm{~ms}, T A S C y c l e=1 \mathrm{~ms}$, and $E S T_{3}=3.5 \mathrm{~ms}$. In the example, $p_{3}$ is the hyperperiod. To minimize the $e 2 e$ time of the corresponding task chain, we normalize the $E S T_{3}$ value to specify in which management unit we have to look for slots first. The first step translates the $E S T_{3}$ value to the hyperperiod: $E S T_{3} \%$ hyperperiod $=1.5 \mathrm{~ms}$. The second step determines the index of the management unit that starts with 1: $\lfloor(1.5 \mathrm{~ms}) / T A S C y c l e\rfloor+1=2$. Hence, we search for free slots in the second management unit on the whole path starting on link $l_{1,2}$ with the offset of $E S T_{3} \% T A S C y c l e=0.5 \mathrm{~ms}$ first. If no slots can be found in the specified management unit, we continue the slot search in the next management unit. The search offset will then be reset to zero. We resume the search until we find suitable slots across the hyperperiod or fail to find a feasible solution. In the course of the scheduling process, the number of occupied slots rises and the number of free slots decreases. Slots of network resources can not be occupied across management units. Still, solutions can be easily found since the network delay of critical streams is small due to high link speeds in time-sensitive networks. This approach also works for multicast streams. The described procedure is applied in the method searchSlotNet in Algorithm 2 Section V-F that receives a stream $\tau_{i}^{n}$ as input. 


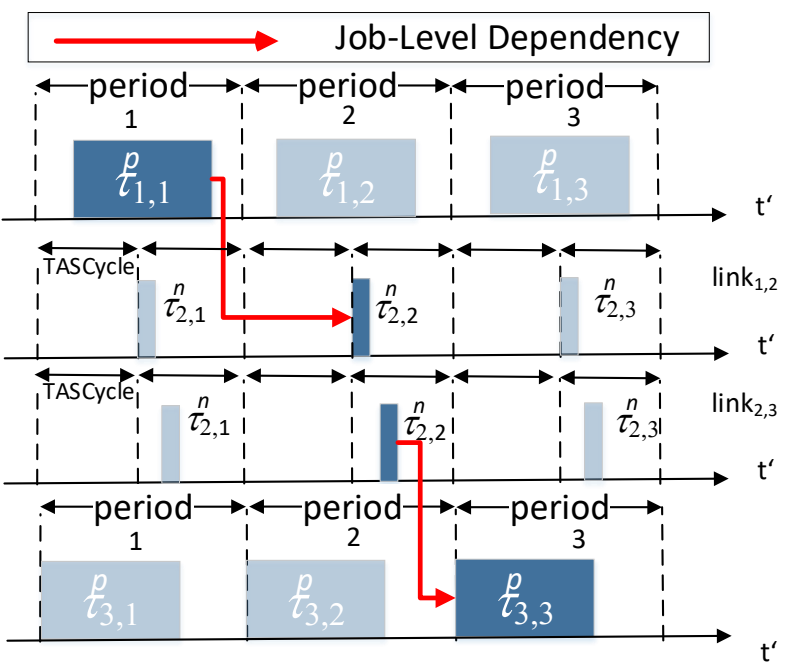

Fig. 11: Example of a CEC schedule.

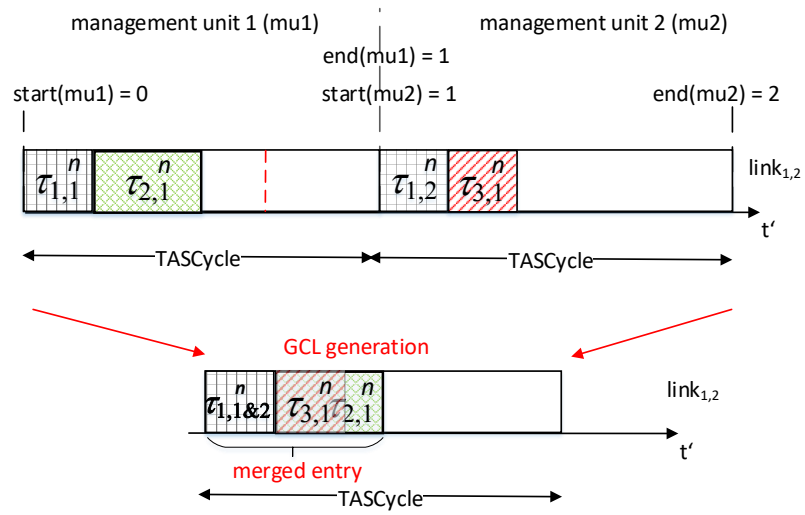

Fig. 12: Example of a GCL merge.

Fig. 11 shows the management of resources and the ratio of the periods applied for the scheduling of processing and network tasks. The assumed network hyperperiod and task period is period. TASCycle is period $/ 2$. The stream $\tau_{2}^{n}$ did not find fitting slots in the first management unit. Thus, the algorithm resumed the slot search in the second management unit and found suitable slots on the links $l_{1,2}$ and $l_{2,3}$.

\section{E. Generation of Gate Control Lists}

The GCLs are generated after the scheduling algorithm has found a feasible solution. The TAS cycle in the GCLs corresponds to TASCycle. After one TASCycle, the GCL schedule is repeated in each TSN-capable egress port or queuing discipline. To construct the GCL, we collect all occupied slots associated with network tasks from all management units of the port and translate the start and end times of the slots to the range of TASCycle by applying the modulo operator. After sorting the translated times in ascending order, we create the GCL entries. First, we assume that each stream gets its own slot. However, we merge two slots if:

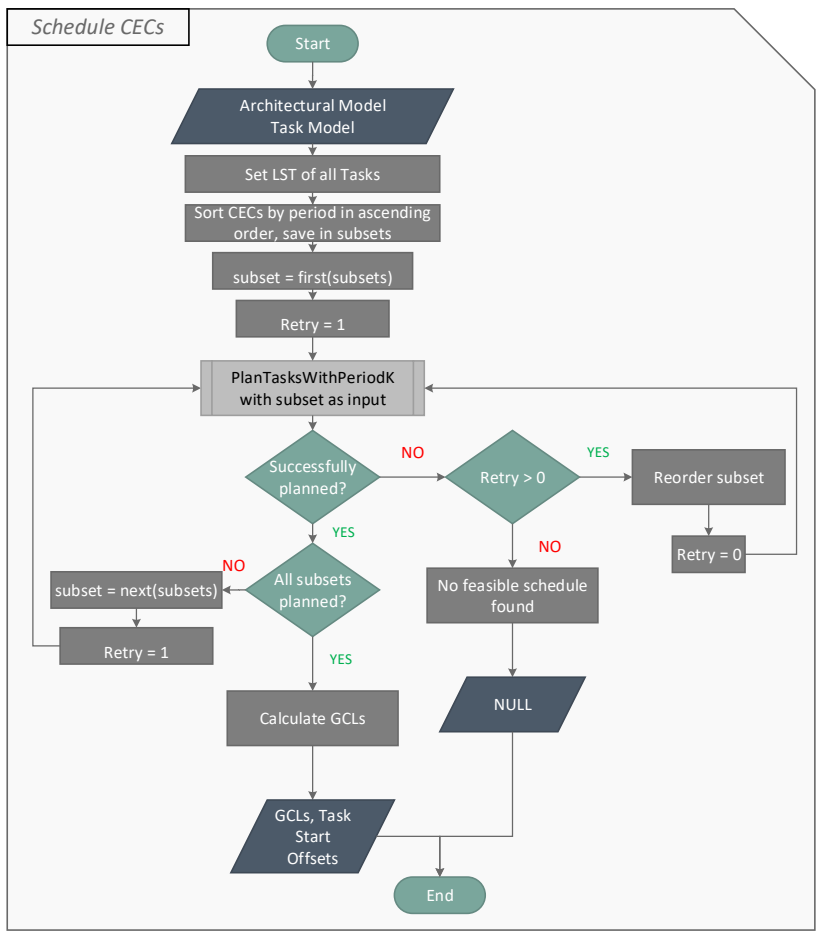

Fig. 13: Overall program flow chart.

- two slots directly follow each other $\left(\tau_{1,1}^{n}\right.$ and $\tau_{2,1}^{n}$ as well as $\tau_{1,2}^{n}$ and $\tau_{3,1}^{n}$ in Fig. 12,

- two slots overlap $\left(\tau_{2,1}^{n}\right.$ and $\tau_{3,1}^{n}$ as well as $\tau_{1,1}^{n}$ and $\tau_{1,2}^{n}$ in Fig. 12,

- two adjacent slots are less apart than the transmission time of the smallest Ethernet frame on wire plus the interframe gap

- not enough entries are available

We will discuss the decision and the advantages of using the TASCycle as the overall network cycle in Section VIII.

\section{F. Overall Scheduling Process}

The proposed scheduling heuristic represents an incremental process that is dependent on the input sequence of CECs. The program flow is illustrated in Fig. 13. We annotate the subset of CECs with a task period $k$ as $\zeta_{k}$. First, we calculate the $L S T$ values for all tasks. Then all CECs are grouped and sorted according to their periods in ascending order. We schedule all subsets $\zeta_{k}$ on the corresponding resources in the function PlanTasksWithPeriodK starting with the smallest $k$. If the method does not find a feasible solution we retry the scheduling of the subset one time by reordering the input subset. On success, we continue planning the subset with the next higher period. If we fail to plan the subset after one retry, we fail the overall scheduling process. Otherwise, if all subsets are planned, we return the GCLs of TAS-capable egress ports or queuing disciplines (when TAPRIO is used) and the start offsets of the processing and network tasks. Those can be used to configure the schedules in the end-device or switch hardware.

Algorithm 1 describes how a subset $\zeta_{k}$ is scheduled. The first step of the function PlanTasksWithPeriodK is the 


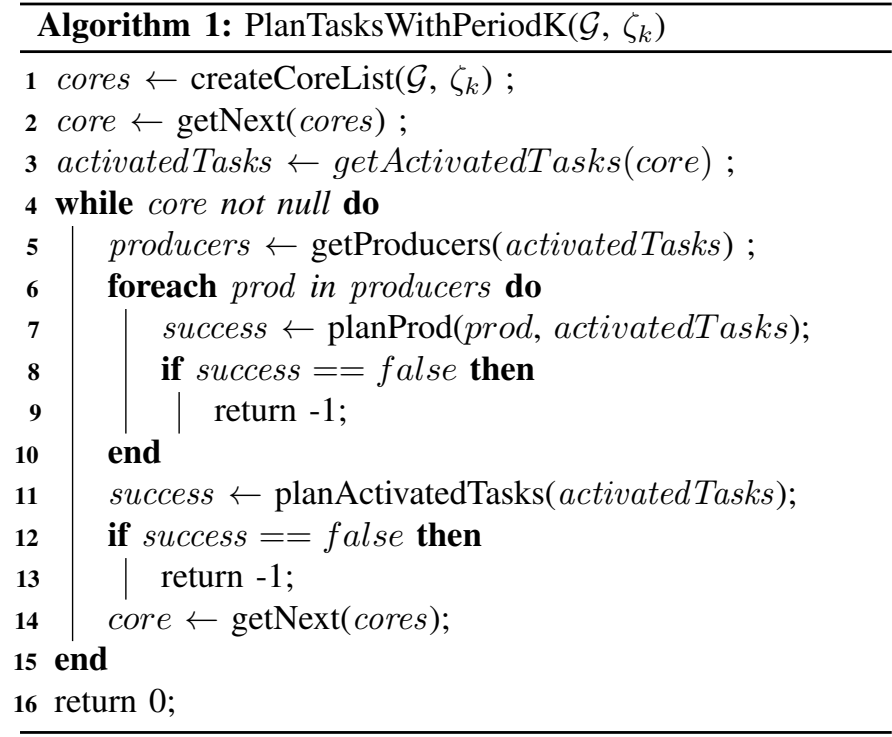

generation of a list of cores that contains all cores used by the producer tasks of $\zeta_{k}$ (line 1). Each producer task is added to the set activatedTasks of its core. The set contains tasks, whose EST and $L S T$ values are already defined. This primarily accounts for all producer tasks. The successors of the producer follow during the schedule run. While there is a core that has processing tasks in activatedTasks, we try to plan all activated tasks, starting with the producer tasks (line 5-10). The producer tasks are removed from activatedTasks. As long as there are unplanned producer tasks on the core, we plan them in the procedure planProd (line 7). This function searches for suitable time slots throughout the hyperperiod for a given input task using the method slotsSearchProc (see also Section V-C). The slots are suitable if they do not make any tasks from activatedTasks miss their LST. If the computed end time of the producer exceeds any $L S T$ value, as allustrated in Fig. 14, the producer task releases its slots and the concerned tasks from activatedTasks are planned first applying slotSearchProc. When a task from activatedTasks is successfully planned, the according slots are occupied and the task is removed from activatedTasks. The function ActivateSuccessorTasks (Algorithm 2) is called in planProd after a successful slot occupation by the activated tasks or the producer task to schedule potentially following streams and to append processing successor tasks of the planned task to activatedTasks of their assigned cores. This validation and searching process continues until the producer task finds suitable slots or until the process fails. We also integrate repair functions that are detailed in Section V-G. Tasks in activatedTasks have predecessors and possibly successors. Consequently, they may be tightly bound by $E S T$ and $L S T$ values in contrast to producer tasks. The rescheduling and fitting of the producer tasks leads to urgent intermediate or last tasks in task chains being planned preferentially, at the same time reducing gaps in the schedule whenever possible. It also makes use of the mobility defined by the difference of $L S T$ and EST.

If all producer tasks can be planned successfully or if there
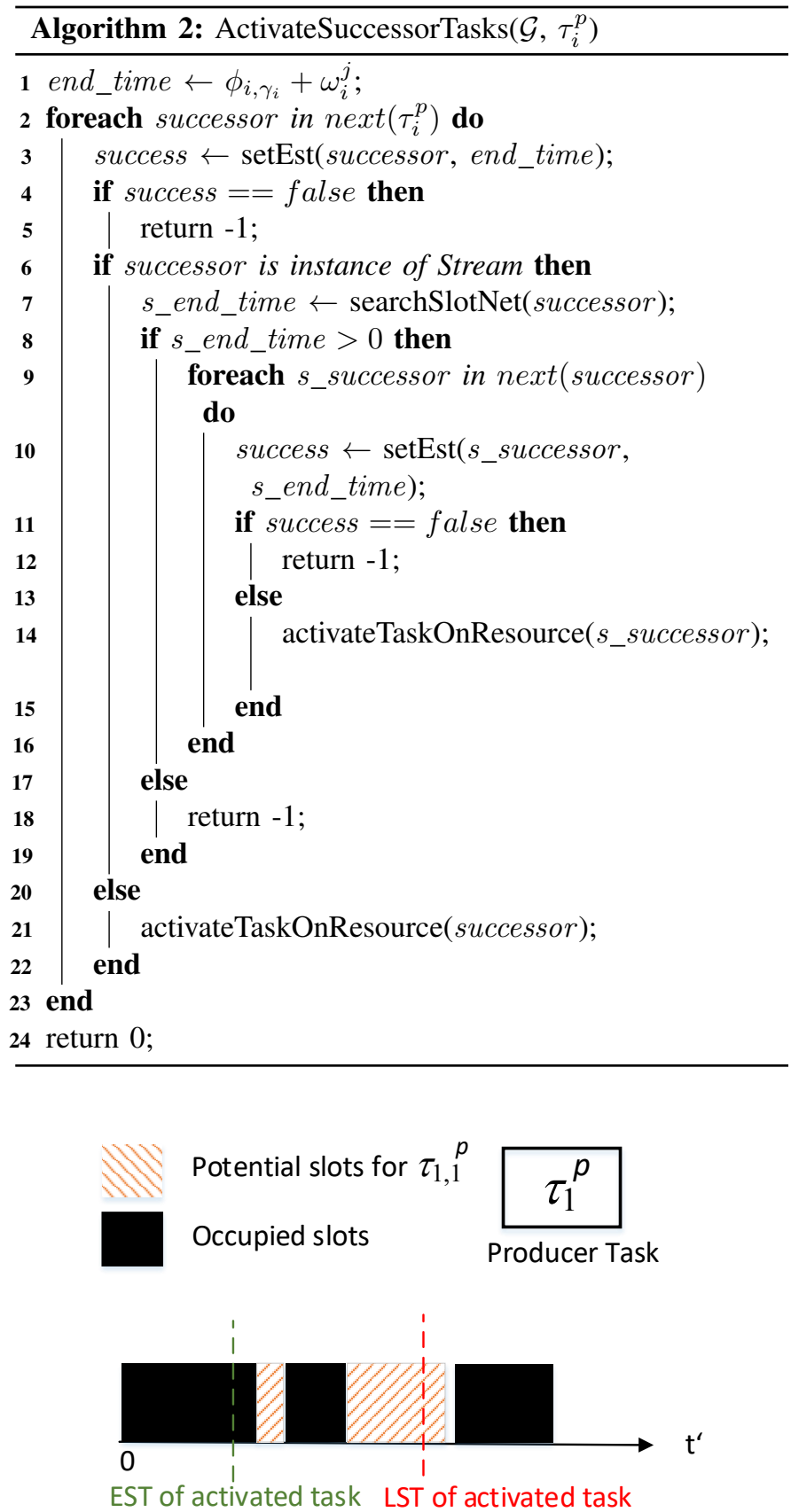

Fig. 14: Job $\tau_{1,1}^{p}$ exceeds the $L S T$ of an activated task.

are no producer tasks on the investigated core, all available tasks in activatedTasks will be planned (line 11). We apply the slotSearch function for each activated task. The tasks in activatedTasks are sorted in ascending order by the EST first and then by the mobility that results from the difference of $E S T$ and $L S T$. We plan task by task. On success, we call ActivateSuccessorTasks. In case of missed deadlines, we apply a repair routine.

Algorithm 2 describes how the successor tasks of a successfully planned task $\tau_{i}^{p}$ are configured and activated in the method ActivateSuccessorTasks. First, we determine the end time of the job $\tau_{i, \gamma_{i}}^{p}$ that refers to the task execution within the job-level dependency. For each successor, we set the 


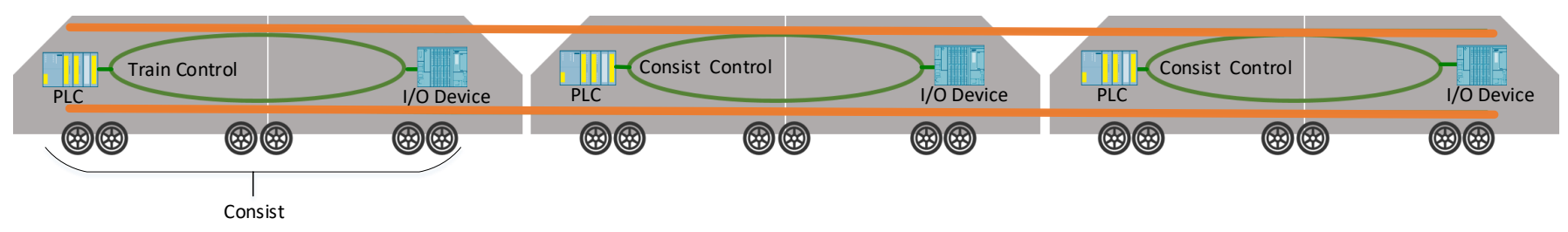

Fig. 15: Simplified train network architecture of the next generation of trains, cf. [33]

$E S T$ bound regarding the end time of $\tau_{i, \gamma_{i}}^{p}$ and the occurring overhead delays (line 3). If the EST is bigger than the $L S T$ the method ActivateSuccessorTasks reports an unsuccessful process. If the successor task of $\tau_{i}^{p}$ is a stream (line 6), then we plan the stream in the method searchSlotNet (see Section V-D. The function searchSlotNet sets the transmission offset, WCTT, and cycle offset and returns the end time of the stream job within the job-level dependency relative to $t^{\prime}=0$ (line 7). After, we set the EST bounds of each stream successor and activate them on their core by adding them to activatedTasks (line 9-16). The activation is done only when all predecessors of the concerned successor task have already been planned.

If the successor of $\tau_{i}^{p}$ is not a stream, i.e. it is a processing task, then we try to activate it on its core (line 21).

\section{G. Repair Functions}

Whenever a processing task or a network task cannot be planned or the $E S T$ value exceeds the $L S T$ value, we apply a repair function in planProd and planActivatedTasks. This includes the following steps:

1) Release all occupied time slots of the according CEC

2) Replan the task chain up to including the failed task. Therefore, align the slot search offset of the producer task $\tau_{a}^{p}: E S T_{a}=\left(E S T_{a}+\frac{1}{4} p_{a}\right) \% p_{a}$

The new specified offset for the producer task is empirical. It showed the best results for our test cases. We allow to repeat this repair method 3 times until we reach the same start offset. If it still fails, then the schedule fails for the subset $\zeta_{k}$. Whenever the schedule fails for the subset $\zeta_{k}$, it is possible to reschedule the subset with another input order of CECs, as already denoted in Fig. 13 More than one retry did not lead to feasible solutions in the majority of our trials.

\section{EXPERIMENTAL RESULTS}

In this section, we evaluate the proposed ITANS heuristic by alternating different parameters such as targeted processor utilization, number of CECs, length of CECs, number of devices, and different task periods. Additionally, we examine our approach with tight and loose end-to-end bounds. One of the pursued metric is the success rate that describes the ratio of execution runs with feasible schedules to execution runs returning infeasible schedules. The other metric is the runtime of the algorithm.

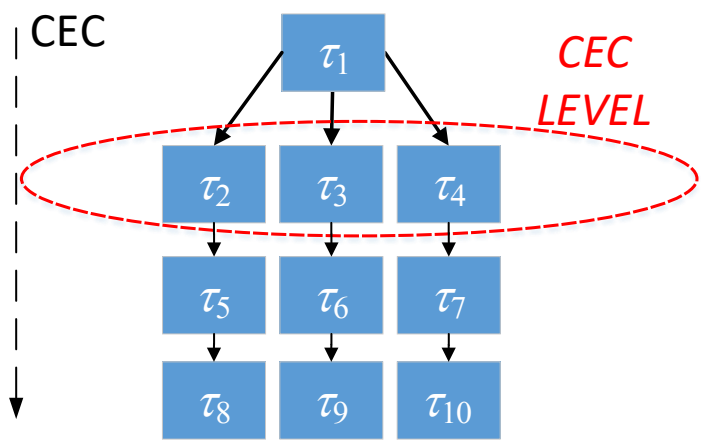

Fig. 16: Multiple tasks on one CEC level.

\section{A. Case Study}

The main emphasis of this case study is to evaluate the usability and performance of our approach. In the context of automated driving and assisted driving, CECs with many CEC levels are more common. Tasks that have the same degree of relationship referred to the producer task are located on the same CEC level (Fig. 16). The high number of CEC levels in the context of automated or assisted driving results from the complex processing chain that includes among others the following steps as stated and detailed in [34]:

- sensing and fusing sensor data

- localization

- interpretation and generation of a world model

- trajectory calculation

- reactive control

- diagnosis and fault management

- trajectory execution

- platform stabilization

In the railway domain, typical use cases are dominated by CECs with few CEC levels but with a high number of tasks on one level, as shown in Fig. 16. This results from the train architecture, see Fig. 15. Signals generated in the main train control application (in the foremost consist) have to be propagated to several consist controls. Then they are processed and distributed to actuators directly or to $\mathrm{I} / \mathrm{O}$ devices that e.g. generate mechanical or electrical signals for actuators. A consist is a compound of one or several railroad cars that contains a closed network with hardware components like controller devices (PLCs), I/O devices, network switches, as well as sensors and actuators. A typical network topology in a consist is a ring topology [35], which is represented by the green circles in Fig. 15. The consist networks are interconnected by redundant line topologies [36] symbolized by orange lines. 
TABLE I: Description of experiments without network tasks

TABLE II: Average number of tasks over all experiments

\begin{tabular}{l|l}
\hline Experiment & Description \\
\hline TightLong & $\begin{array}{l}\text { Tight end-to-end deadline with 2-10 CEC levels, } \\
1 \text { task on each CEC level }\end{array}$ \\
\hline LooseLong & $\begin{array}{l}\text { Less tight end-to-end deadline with 2-10 CEC levels, } \\
1 \text { task on each CEC level }\end{array}$ \\
\hline TightMiddle & $\begin{array}{l}\text { Tight end-to-end deadline with 2-6 CEC levels, } \\
1 \text { task on each CEC level }\end{array}$ \\
\hline TightShort & $\begin{array}{l}\text { Tight end-to-end deadline with 2-4 CEC levels, } \\
1-3 \text { tasks on each CEC level }\end{array}$ \\
\hline
\end{tabular}

\begin{tabular}{l|l|l|l}
\hline \# CECs & \# proc. tasks & \# net. tasks & Total number of tasks \\
\hline 5 & 27 & 18 & 45 \\
\hline 10 & 60 & 32 & 92 \\
\hline 20 & 131 & 50 & 181 \\
\hline 50 & 280 & 108 & 388 \\
\hline 100 & 595 & 228 & 823 \\
\hline 120 & 785 & 280 & 1065 \\
\hline 150 & 916 & 314 & 1230 \\
\hline
\end{tabular}

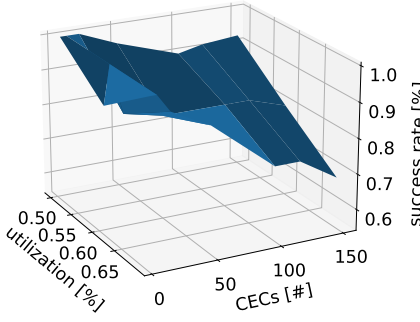

(a) TightLong

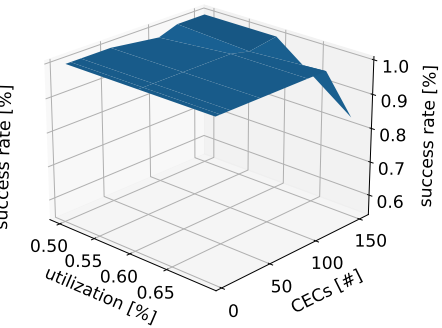

(b) LooseLong

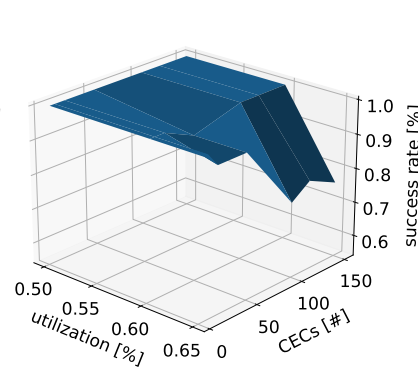

(c) TightMiddle

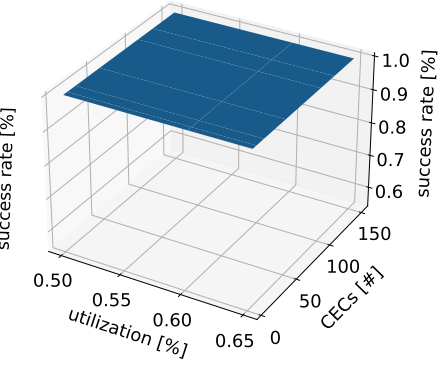

(d) TightShort

Fig. 17: Success rate in comparison to the utilization of processing cores and number of CECs in different experiments.

The orange and green lines represent Ethernet-based links in our example. Ethernet technology is already extensively used in the railway domain today. A train communication network has a dynamic size as further vehicles or whole trains can be coupled or decoupled. This dynamic size influences the number of tasks on a CEC level.

Based on these use cases, we carry out the case study with synthetic tasks and networks varying the following parameters:

- Processing core utilization $\in$ $\{0.5-0.55,0.6-0.65,0.65-0.7\}$

- Number of CECs $\in\{5,10,20,50,100,120,150\}$

- Number of CEC levels and number of tasks on one level

- Periods of the tasks $\in\{2,4,8,16,32,64,128\} \mathrm{ms}$

- Number of switches in the network $\in\{3,5,10,20,30\}$

The number of end-devices depends on the utilization. The number of processing cores was randomly chosen between one and two cores per end-device, where predominantly one core was selected to force the distribution of tasks across the system. Each task was randomly assigned to one processing core. On average, the number of generated end-devices was slightly smaller than the number of task chains. Whenever two subsequent tasks of a chain were distributed across different end-devices, a communication task was inserted. The assumed WCET of a processing task was less than or equal to $20 \%$ of its period and smaller than $30 \%$ of the smallest period on the same core. The heuristic was implemented and tested on a Core i7-8550U CPU, $1.88 \mathrm{GHz}$ processor with 24GB RAM.

As described in Table I] we carry out 4 experiments and vary the parameters mentioned previously. Table I defines the tightness of the end-to-end deadlines, the number of CEC levels, and the number of tasks on one CEC level. The network tasks are excluded in Table I. They will be added after task allocation and they will contribute to more CEC levels and more tasks on a level. For our experiments, we define tight end-to-end bounds for job-level dependencies relying on WCETs and WCTTs of the tasks in a task chain. Therefore, we sum up the WCETs and WCTTs of the processing and network tasks of a CEC and add a mobility interval of 30$50 \%$ of the homogeneous task period. Then we round it up to the next larger multiple of the task period. For example: If the period of all tasks of a CEC is $1 \mathrm{~ms}$ and the sum of WCETs, WCTTs, and the mobility interval results in $1.2 \mathrm{~ms}$, we set the end-to-end deadline of the job-level dependency to the next multiple of the period, which results in $2 \mathrm{~ms}$. The experiment with less tight (loose) bounds increases the mobility interval to $60-80 \%$ of the period.

\section{B. Results}

In the first step, we have determined the success rate in dependence of the processor utilization and the amount of CECs. Therefore, we have fixed the number of switches to 20 switches. The chosen network topology in the following experiments was a meshed topology. The average number of processing and network tasks in relation to the number of CECs is depicted in Table II. We have executed each possible configuration (utilization, number of CECs) around 30 times and we have taken the average result. The experiment TightLong (Fig. 17a) shows a high success rate for the utilization of $0.5-0.55$. The success rate decreases with higher utilization and number of CECs. Tight end-to-end bounds lead to a smaller difference of the EST and $L S T$ values of tasks and thus to less flexibility in task planning. Fig. 17b shows the outcome of the test LooseLong. The results demonstrate a success rate of over $80 \%$, while the experiment TightMiddle Fig. 17c exhibits a success rate of over $75 \%$. Short CECs with several tasks on one level provide the best results in TightShort, see 


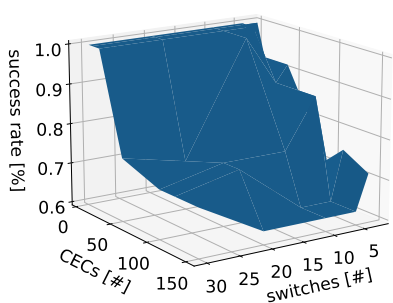

(a) TightLong

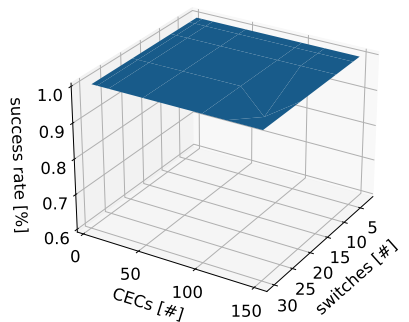

(b) LooseLong
Fig. 18: Success rate in comparison to the number of switches and the number of CECs for different experiments.

Fig. 17d It is evident that the number of CECs and the number of CEC levels have the most impact on the success rate. The test TightShort has the same number of processing tasks as TightLong, but it provides a higher success rate. This can be traced back to fewer CEC levels in TightShort.

Furthermore, we set the number of switches, the number of CECs, and the success rate in relation. The utilization rate was fixed at 0.6-0.65. We have executed each configuration (number of switches, number of CECs) around 30 times and we have taken the average. If we take a closer look at the impact of the number of switches in the system, we see in Fig. 18a and Fig. 18b that the success rate particularly drops with the number of CECs. We cannot detect a clear trend for the influence of the number of switches in Fig. 18a. The experiment LooseLong (Fig. 18b) provides a high success rate in general. The rate slightly drops for 30 switches. Logically, a small number of switches might transfer more network traffic per switch and per egress port and might lead to more conflict situations. At the same time, less switches on a stream path reduce the WCTT. On the other hand, longer stream paths due to a higher number of switches increase the WCTTs of network tasks. Nevertheless, more switches are able to promote a better balancing of network load and modern link speeds favor a fast transmission through the network.

We illustrate the runtime of the ITANS heuristic in Fig. 19. Therefore, we have recorded the average execution time of successful runs varying the number of CECs and the processor utilization fixing the number of switches in the system. The most influencing factor is the number of CECs. The runtime increases linearly with the number of CECs The line plots for different numbers of switches deviate only slightly. We are able to find feasible solutions for more than 1000 network and processing tasks in under $3 \mathrm{~s}$.

\section{Schedule Plausibility}

To check the plausibility of the resulting schedules, we have simulated the runs and compared them to the resulting schedules. Therefore, we have performed the following steps:

- examination of the schedules of each task chain and its deadline

- evaluation of each stream schedule from the talker node to each listener

- investigation of the complete schedule on each traversed network link

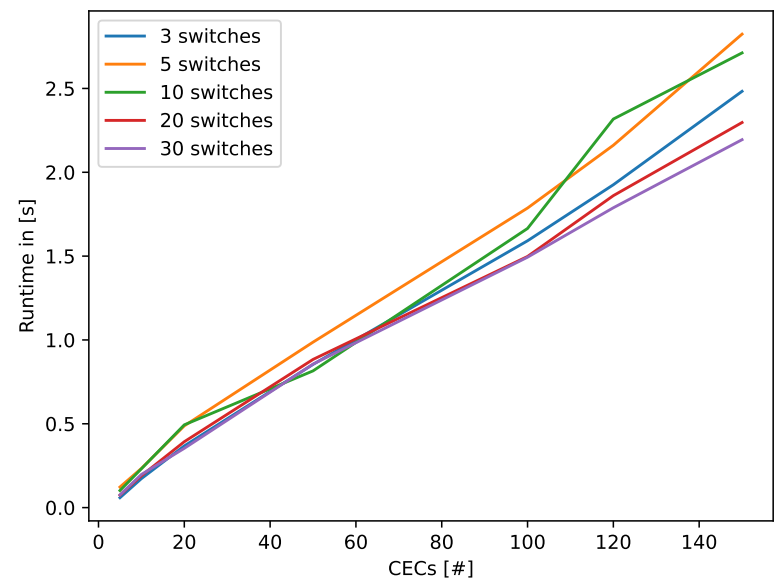

Fig. 19: Runtime of ITANS.

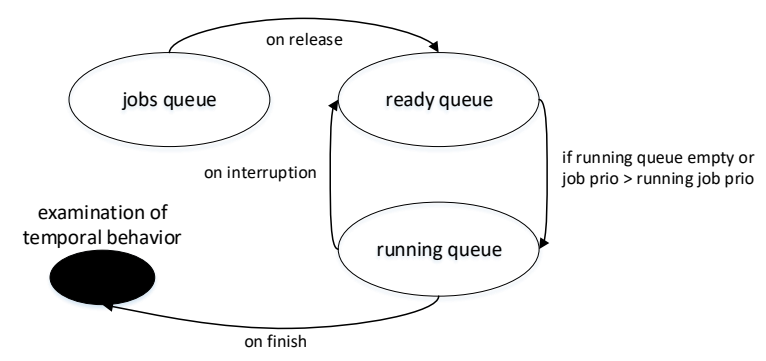

Fig. 20: Visualization of scheduling states on a core.

- validation of the schedules on each processing core

To ensure that the resulting end-to-end boundaries are compliant with the required ones, we create the job-level dependencies of each CEC and calculate the end-to-end times from the obtained release time of the first job in the joblevel dependency until the completion time of the last jobs, as already illustrated in Fig. 3

To verify the processing task schedule, we construct a priority-based schedule on each processing core. Therefore, we create jobs with the calculated release times and WCRTs for all available tasks on the considered core throughout two hyperperiods and sort them by the release times (ascending order). We have to exceed the consideration beyond one hyperperiod because we allow tasks to start in one cycle and finish in the subsequent cycle. The high-level view of this validation process is presented in Fig. 20 Those jobs are initially managed in a jobs queue. The evaluation jumps from event to event to generate a time advance. Such an event is represented by the following steps:

- release of a job

- start or continuation of the execution of a job

- interruption of a running job

- completion of a running job

We start at the earliest release time of a job. Whenever a job is released, i.e. it leaves the jobs queue and enters the ready queue, we check if there is a job in the running queue. The running queue holds one job maximally. If not, we dequeue the job with the highest priority from the ready queue and put it in the running queue. If there is a running job and 


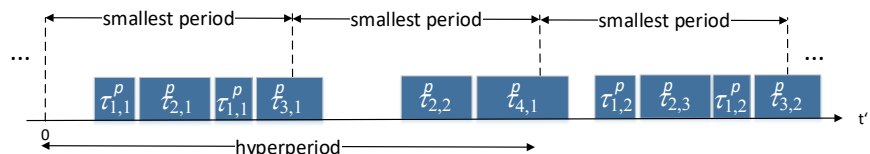

Fig. 21: Visualization of a processing task schedule on a core.

its priority is lower, we interrupt the running job, dequeue it from the running queue and put it in the ready queue before running the ready job that has a higher priority. Whenever a running job finishes, we compare the simulated WCRT with the calculated one that resulted from our schedule run. The schedule is infeasible if the calculated WCRT of our approach differs from the simulated one. After the comparison, we either run the job with the highest priority in the ready queue or, if there are no jobs in the ready queue, we jump to the release time of the first job in the jobs queue. We take into account the scheduling delays in our simulation. An exemplary schedule is illustrated in Fig. 21

To evaluate each stream schedule, we first determine the static delays between each two adjacent links on the stream path. Then we calculate the expected arriving times and WCTTs from the talker node to each listener node. We delay the simulated results by the calculated release offset of the stream. If the simulated values and the output values of the schedule run do not match, then the plan is infeasible.

In addition, we check if any streams overlap on any egress port in the system. This means that the transmission of one stream shall not start before the previous one has left the port and before the elapse of the interframe gap time.

The plausibility checks have shown that the simulated schedules have matched with the calculated schedules in all runs.

\section{RELATED WORK}

Scheduling of CECs combines different research areas. We want to address the related work that deals with the scheduling of TSN streams applying the TAS mechanism, joint task and network planning, and CECs. In the field of stream planning in time-sensitive networks, different scheduling approaches have arisen. Craciunas et al. [28] formulate scheduling constraints for 802.1Qbv-compliant networks and introduce the stream isolation principle to avoid dynamic network delays such as the queuing delay in switch buffers. They solve the problem of scheduling of up to 1000 TSN streams with predetermined paths by using Satisfiability Modulo Theories (SMT) and Optimization Modulo Theories (OMT). The approaches presented in [29], [37] apply genetic algorithms to schedule timetriggered traffic in time-sensitive networks. Dürr et al. [38] present a mapping of the Job Shop Scheduling Problem (JSP) to the planning of TSN traffic. They create stream schedules by integrating the stream isolation method and the Tabu Search principle. In [39], a window-based approach that determines the GCL entries is presented. The authors embed a worstcase delay analysis to guarantee upper delay bounds instead of using strict temporal isolation. Our approach for creating stream schedules includes a window-based search as well but also integrates the stream isolation principle. As we combine the task and network schedule, we employ an incremental firstfit strategy. In comparison to the discussed works, we show a low runtime but do not further optimize the stream schedules.

Regarding combined task and network scheduling strategies, Zhang et al. [26] propose a Mixed Integer Programming (MIP)-based approach for non-preemptive periodic applications and network tasks. They define the objectives to optimize the end-to-end task chain latencies and response times of applications. They are able to find optimal solutions for small until middle-sized industrial use cases with up to 90 task chains consisting of 2 processing and one network task in less than $150 \mathrm{~s}$. The SMT-based approach presented in [40] solves the combined task and network scheduling problem for middle- to large-sized industrial use cases. The runtime of the SMT-based variant varies from a few milliseconds to several hours. The authors focus on the earliest deadline first (EDF) scheduling approach for preemptive processing tasks. They formulate network, task, and memory constraints. In contrast, the work presented in [26] and the proposed ITANS heuristic neglect the memory constraints. We put the emphasis on middle- to large-sized problems with respect to TSN-based communication networks integrating the $802.1 \mathrm{Qbv}$ mechanism. [26], [40] focus on time-triggered Ethernet networks using protocols like TTEthernet. As already mentioned, we present an algorithm that is able to find feasible solutions for the combined planning of processing and network tasks in a low runtime considering end-to-end latencies for CECs. We apply a fixed-priority schedule and allow the preemption of processing tasks.

The CEC topic is also well researched. Kramer et al. [41] provide automotive benchmarks taking typical CEC characteristics into consideration. They present among others common task periods and execution times of tasks in the automotive field. Becker et al. [42] introduce an end-to-end response time analysis for CECs in automotive embedded systems. They focus on the computation of the maximum data age of CECs. The ITANS heuristic works the other way around. We try to schedule the tasks of the CECs so that we comply to given end-to-end times. Schlatow et al. [43] propose a Mixed Integer Linear Program (MILP)-based optimization for the data age of CECs comprising preemptive processing tasks. They determine the phase offset, priority, and processor mapping of processing tasks minimizing the data age. Network tasks are not considered in this work. The authors show that their approach is applicable to real work automotive use cases such as Advanced Driver Assistance Systems (ADAS) and engine control.

We mainly put the emphasis on finding feasible solutions for different system constellations using Ethernet-based networks with the TAS mechanism in a short time. Therefore, we account for different application domains and use cases. The proposed approach is especially applicable during the system design, the test period (e.g. for system simulations [30]), reconfiguration scenarios, and also for the actual operating mode of the system. Exact algorithms or meta-heuristics can lead to long runtimes resulting in hours or days. Nevertheless, it must be mentioned that the resulting solutions of heuristic 
approaches are often not optimal and only a part of the solution space is explored.

\section{DISCUSSION AND CONCLUSION}

We have shown that it is possible to calculate feasible schedules for time-triggered processing and network tasks with different requirements. The methods were chosen to satisfy both. Other approaches are also plausible. It is e.g. conceivable that first all processing tasks of the CECs are planned and then a feasible schedule for the network has to be found. However, in this case the release times of succeeding processing tasks on different end-devices have to be estimated as the intermediate network task will be scheduled later. Moreover, this approach still requires a worst-case estimation of network delays, e.g. through analytical methods like network calculus, to determine the earliest start time of the succeeding processing tasks on different end-devices. If the network delay is underestimated data will be delivered to a later execution of the succeeding processing task and data age will rise. It is especially difficult to meet the end-to-end deadlines of the CECs when regarding both separately. An incremental and combined approach allows to better synchronize the processing and network tasks and to find less pessimistic bounds for network latency and jitter when applying the TAS mechanism.

The ITANS approach involves the use of static priorities for processing tasks. Dynamic priorities, as e.g. used for scheduling policies like EDF, might reorder already scheduled tasks in our approach. This can lead to the tasks no longer being coordinated with other tasks in the task chain and to missing the end-to-end deadline.

EDF and RM scheduling can achieve $100 \%$ core utilization when using harmonic tasks [44]. Yet, it is difficult to achieve this utilization for CECs, especially when release offsets and tight end-to-end deadlines are involved. Therefore, we account for some slack times.

Moreover, we reduce the selection of periods to harmonic periods. Harmonic task periods that are used in our approach are common in many application domains [44]. However, it is not always possible to exclusively use harmonic task periods, as the definition of periods strongly depends on the sensors and actuators. E.g. camera sensors provide certain sample rates that might not harmonize with the sample rate of other sensors such as LiDAR or radar.

Also, we have assumed that the task periods in a CEC are homogeneous. As stated in [41], this applies to $70 \%$ of engine control applications as can be found in the automotive, industrial automation, and railway domain. On the other hand, if we assumed harmonic periods and allowed different periods among CECs, we would lose or waste signals if the successor tasks had a higher period [45] due to over- or undersampling. Consequently, we still cover many use cases.

Nonetheless, we have to annotate that we neglect the consideration of memory resources in our approach. This concerns memory that is necessary for the execution of processing tasks and the buffers for network traffic. Among others, there are buffer restrictions in the NICs of end-devices and in the traffic control module, if it is available in the OS. The difference between the best-case execution time (BCET) and WCET of processing tasks leads to network packets arriving at traffic control modules or NICs earlier than the computed and configured forwarding time. Thus, early packets have to be buffered for some time. Also when network packets arrive at network switches and before they are processed and forwarded to the proper egress ports, they have to be buffered. Depending on the number of ingress ports this can be more or less critical. The memory consumption due to queuing in egress ports is in our case not of interest as we avoid queuing delays.

We do not elaborate on the problem of shared or exclusive resources for processing tasks that is referenced extensively in [46]. We leave the analysis to system or application designers. Nevertheless, we can integrate those times in the WCRTs. Also, the clock drift between clocks of different devices was not considered. Synchronized clocks are necessary for the TAS mechanisms and the CEC task sequence. We have assumed ideal clocks. The PTP protocol and adaptations of it are common time synchronization protocols for Ethernetbased systems that promise a maximum deviation in the submicrosecond range between clocks. This deviation can be considered in the proposed formulas.

In Section V-D, we have introduced the TASCycle that represents the smallest period of all network tasks. Planning with the smallest period reduces the number of entries in the GCL of a TSN-capable egress port. The maximum number of GCL entries is limited. Streams with a greater period than $T A S C y c l e$ can occupy one slot alternatively if they use different TASCycles or management units. However, if the alternation is not optimal, the whole slot or a part of the slot will be reserved in every $T A S C y c l e$, even though it is not used. On the other side, if we constructed the GCLs using the hyperperiod and if the difference between the biggest and the smallest stream period in the system were large, the number of necessary entries in the GCL could exceed the number of maximum allowed entries in the hardware. In this case, entries would have to be merged and bandwidth would also be wasted. Moreover, the configuration would become less manageable due to many entries.

In summary, we presented a heuristic approach for the joint task and network scheduling covering common problems in a variety of application domains. Furthermore, we have shown that our algorithm is capable to find feasible solutions for middle- to large-sized problems in just a few seconds. The ITANS heuristic is well suited for reconfiguration scenarios, which are caused, for example, by changes to the software or hardware. This scenario is common in the railway domain when coupling or decoupling trains or railroad cars. The runtime of the ITANS algorithm promises fast results and avoids long downtimes. In the future, we plan to elaborate the algorithm by including meta-heuristics like genetic algorithms to determine a more optimal input order of CECs. Additionally, we want to extend our approach to non-harmonic periods and multi-rate periods within task chains. We are currently developing simulation setups and evaluations for CECs in the simulation environment OMNeT++. We will examine the proposed schedule in more detail with simulation environments and real hardware in the next steps. 


\section{REFERENCES}

[1] C. Mannweiler, B. Gajic, P. Rost, R. S. Ganesan, C. Markwart, R. Halfmann, J. Gebert, and A. Wich, "Reliable and Deterministic Mobile Communications for Industry 4.0: Key Challenges and Solutions for the Integration of the 3GPP 5G System with IEEE," in Mobile Communication - Technologies and Applications; 24. ITG-Symposium, 2019, pp. 1-6.

[2] L. Silva, P. Pedreiras, P. Fonseca, and L. Almeida, "On the adequacy of SDN and TSN for Industry 4.0," in 2019 IEEE 22nd International Symposium on Real-Time Distributed Computing (ISORC), 2019, pp. 43-51.

[3] Z. Wang, Y. Wu, and Q. Niu, "Multi-sensor fusion in automated driving: A survey," IEEE Access, vol. 8, pp. 2847-2868, 2020.

[4] R. Lagay and G. M. Adell, "The Autonomous Train: a game changer for the railways industry," in 2018 16th International Conference on Intelligent Transportation Systems Telecommunications (ITST), 2018, pp. $1-5$.

[5] Time-Sensitive Networking (TSN) Task Group. [Online]. Available: https://1.ieee802.org/tsn/

[6] N. Finn, IEEE Communications Standards Magazine.

[7] J. L. Messenger, "Time-Sensitive Networking: An Introduction," IEEE Communications Standards Magazine, vol. 2, no. 2, pp. 29-33, 2018.

[8] F. Zezulka, P. Marcon, Z. Bradac, J. Arm, T. Benesl, and I. Vesely, "Communication Systems for Industry 4.0 and the IIoT," IFACPapersOnLine, vol. 51, no. 6, pp. 150-155, 2018, 15th IFAC Conference on Programmable Devices and Embedded Systems PDeS 2018.

[9] S. Brunner, J. Roder, M. Kucera, and T. Waas, "Automotive E/Earchitecture enhancements by usage of ethernet TSN," in 2017 13th Workshop on Intelligent Solutions in Embedded Systems (WISES), 2017. pp. 9-13.

[10] H. Fang and R. Obermaisser, "Execution Environment for MixedCriticality Train Applications Based on an Integrated Architecture," in 2017 International Conference on Promising Electronic Technologies (ICPET), 2017, pp. 1-7.

[11] S. Aust, "Paving the Way for Connected Cars with Adaptive AUTOSAR and AGL," in 2018 IEEE 43rd Conference on Local Computer Networks Workshops (LCN Workshops), 2018, pp. 53-58.

[12] S. Fürst and M. Bechter, "AUTOSAR for Connected and Autonomous Vehicles: The AUTOSAR Adaptive Platform," in 2016 46th Annual IEEE/IFIP International Conference on Dependable Systems and Networks Workshop (DSN-W), Jun. 2016, pp. 215-217.

[13] A. Arestova, M. Martin, K.-S. J. Hielscher, and R. German, "A ServiceOriented Real-Time Communication Scheme for AUTOSAR Adaptive Using OPC UA and Time-Sensitive Networking," Sensors, vol. 21, no. 7, 2021. [Online]. Available: https://www.mdpi.com/1424-8220/21/7/2337

[14] M. Günzel, K.-H. Chen, N. Ueter, G. v. d. Brüggen, M. Dürr, and J.J. Chen, "Timing Analysis of Asynchronized Distributed Cause-Effect Chains," in IEEE Real-Time and Embedded Technology and Applications Symposium (RTAS), 2021.

[15] IEEE Computer Society, "IEEE Standard for Local and Metropolitan Area Networks - Bridges and Bridged Networks - Amendment 26: Frame Preemption," IEEE Std 802.1Qbu-2016 (Amendment to IEEE Std 802.1Q-2014), Aug. 2016.

[16] "IEEE Standard for Local and metropolitan area networks-Bridges and Bridged Networks-Amendment 29: Cyclic Queuing and Forwarding," IEEE 802.1Qch-2017 (Amendment to IEEE Std 802.1Q-2014 as amended by IEEE Std 802.1Qca-2015, IEEE Std 802.1Qcd(TM)-2015, IEEE Std 802.1Q-2014/Cor 1-2015, IEEE Std 802.1Qbv-2015, IEEE Std 802.1Qbu-2016, IEEE Std 802.1Qbz-2016, and IEEE Std 802.1Qci2017), pp. 1-30, 2017.

[17] "IEEE Standard for Local and Metropolitan Area Networks-Bridges and Bridged Networks - Amendment 34:Asynchronous Traffic Shaping," IEEE Std 802.1Qcr-2020 (Amendment to IEEE Std 802.1Q-2018 as amended by IEEE Std 802.1Qcp-2018, IEEE Std 802.1Qcc-2018, IEEE Std 802.1Qcy-2019, and IEEE Std 802.1Qcx-2020), pp. 1-151, 2020.

[18] IEEE Computer Society, "IEEE Standard for Local and Metropolitan Area Networks - Bridges and Bridged Networks - Amendment 25: Enhancements for Scheduled Traffic," IEEE Std 802.1Qbv-2015 (Amendment to IEEE Std 802.1Q-2014 as amended by IEEE Std 802.1Qca-2015, IEEE Std 802.1Qcd-2015, and IEEE Std 802.1Q-2014/Cor 1-2015), Mar. 2016.

[19] $\_$, "IEEE Standard for Local and Metropolitan Area NetworksTiming and Synchronization for Time-Sensitive Applications," IEEE Std 802.1AS-2020 (Revision of IEEE Std 802.1AS-2011), 2020.

[20] "tc(8) - Linux manual page," https://man7.org/linux/man-pages/man8/ tc.8.html accessed: 2021-09-16.
[21] "tc-etf(8) - Linux manual page," https://man7.org/linux/man-pages/ man8/tc-etf.8.html accessed: 2021-08-16.

[22] "tc-mqprio(8) — Linux manual page," https://man7.org/linux/ man-pages/man8/tc-mqprio.8.html accessed: 2021-08-16.

[23] "tc-taprio(8) — Linux manual page," https://man7.org/linux/man-pages/ man8/tc-taprio.8.html accessed: 2021-08-16.

[24] "Scheduled packet Transmission: ETF," https://lwn.net/Articles/758592/ accessed: 2021-09-16.

[25] C. S. V. Gutiérrez, L. U. S. Juan, I. Z. Ugarte, and V. M. Vilches, "Realtime Linux communications: an evaluation of the Linux communication stack for real-time robotic applications," ArXiv, 2018.

[26] L. Zhang, D. Goswami, R. Schneider, and S. Chakraborty, "Task- and network-level schedule co-synthesis of ethernet-based time-triggered systems," Proceedings of the Asia and South Pacific Design Automation Conference, ASP-DAC, pp. 119-124, 012014.

[27] M. Becker, D. Dasari, S. Mubeen, M. Behnam, and T. Nolte, "Synthesizing job-level dependencies for automotive multi-rate effect chains," in 2016 IEEE 22nd International Conference on Embedded and Real-Time Computing Systems and Applications (RTCSA), 2016, pp. 159-169.

[28] S. S. Craciunas, R. S. Oliver, M. Chmelík, and W. Steiner, "Scheduling Real-Time Communication in IEEE 802.1Qbv Time Sensitive Networks," in Proceedings of the 24th International Conference on RealTime Networks and Systems, ser. RTNS '16. New York, NY, USA: ACM, 2016, pp. 183-192.

[29] A. Arestova, K.-S. J. Hielscher, and R. German, "Design of a Hybrid Genetic Algorithm for Time-Sensitive Networking," in Measurement, Modelling and Evaluation of Computing Systems. Springer International Publishing, Mar. 2020, pp. 99-117.

[30] A. Arestova, K.-S. Jens Hielscher, and R. German, "Simulative evaluation of the tsn mechanisms time-aware shaper and frame preemption and their suitability for industrial use cases," in 2021 IFIP Networking Conference (IFIP Networking), 2021, pp. 1-6.

[31] "Industrial communication networks - Profiles - Part 2: Additional fieldbus profiles for realtime networks based on ISO/IEC/IEEE 88023," Norm DIN EN IEC 61784-2, Jul. 2020.

[32] H. Kopetz, A. Ademaj, P. Grillinger, and K. Steinhammer, "The timetriggered Ethernet (TTE) design," in Eighth IEEE International Symposium on Object-Oriented Real-Time Distributed Computing (ISORC'05), May 2005, pp. 22-33.

[33] A. Arriola, M. Münzer, A. Geven, J. Pujol, H. Envall, J. Härri, N. Mata, and R. Aigner, 2020.

[34] S. Behere and M. Törngren, "A functional reference architecture for autonomous driving," Information and Software Technology, vol. 73, pp. 136-150, 2016. [Online]. Available: https://www.sciencedirect.com/ science/article/pii/S0950584915002177

[35] IEC 61375-1:2012. Electronic railway equipment - Train communication network (TCN) - Part 1: General architecture, 3rd ed., Jun. 2012.

[36] IEC 61375-2-5:2014. Electronic railway equipment - Train communication network (TCN) - Part 2-5: Ethernet train backbone, 1st ed., Aug. 2014.

[37] M. Pahlevan and R. Obermaisser, "Genetic Algorithm for Scheduling Time-Triggered Traffic in Time-Sensitive Networks," in 2018 IEEE 23rd International Conference on Emerging Technologies and Factory Automation (ETFA), vol. 1, Sep. 2018, pp. 337-344.

[38] F. Dürr and N. G. Nayak, "No-wait Packet Scheduling for IEEE Timesensitive Networks (TSN)," in Proceedings of the 24th International Conference on Real-Time Networks and Systems, ser. RTNS '16. New York, NY, USA: ACM, 2016, pp. 203-212.

[39] N. Reusch, L. Zhao, S. S. Craciunas, and P. Pop, "Window-based schedule synthesis for industrial ieee 802.1qbv tsn networks," in 2020 16th IEEE International Conference on Factory Communication Systems (WFCS), 2020, pp. 1-4.

[40] S. Craciunas and R. Serna Oliver, "Combined task- and network-level scheduling for distributed time-triggered systems," Real-Time Systems, vol. 52, 032016

[41] S. Kramer, D. Ziegenbein, and A. Hamann, "Real world automotive benchmarks for free," in 6th International Workshop on Analysis Tools and Methodologies for Embedded and Real-time Systems, 2015.

[42] "End-to-end timing analysis of cause-effect chains in automotive embedded systems," Journal of Systems Architecture, vol. 80, pp. 104-113, 2017.

[43] J. Schlatow, M. Mostl, S. Tobuschat, T. Ishigooka, and R. Ernst, "Dataage analysis and optimisation for cause-effect chains in automotive control systems," in 2018 IEEE 13th International Symposium on Industrial Embedded Systems (SIES), 2018, pp. 1-9. 
[44] M. Mohaqeqi, M. Nasri, Y. Xu, A. Cervin, and K. Årzén, "Optimal harmonic period assignment: complexity results and approximation algorithms," Real-Time Systems, vol. 54, pp. 830-860, 2018.

[45] E. Farcas, C. Farcas, W. Pree, and J. Templ, "Transparent distribution of real-time components based on logical execution time," SIGPLAN Not., vol. 40, no. 7, p. 31-39, Jun. 2005.

[46] G. C. Buttazzo, Hard Real-Time Computing Systems: Predictable Scheduling Algorithms and Applications, 3rd ed. Springer Publishing Company, Incorporated, 2011.

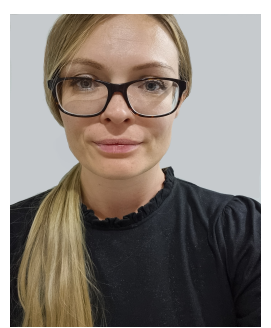

Anna Arestova studied Information and Communication Technology in the bachelor's and master's program at the University of Erlangen-Nürnberg (Germany). She received her M.Sc. degree in 2018. At the moment, she is pursuing the Ph.D. degree at the department of Computer Science at the University of Erlangen-Nürnberg. Her research interests are Time-Sensitive Networking, modelling and simulation of communication networks, as well as real-time scheduling.

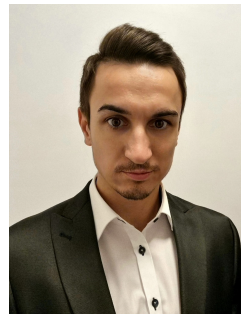

Wojciech Baron received his B.Sc. (2015) and M.Sc. (2018) degree in Information and Communication Technology from the University of ErlangenNürnberg, Germany. At the moment, he is working as a Ph.D. student at the Department of Computer Science at the University of Erlangen-Nürnberg. He collaborates with AUDI AG in diverse projects that evaluate synchronization mechanisms and real-time capabilities in distributed simulation systems in the context of automated driving.

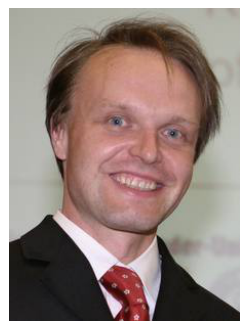

Kai-Steffen J. Hielscher was born in Münchberg, Germany, in 1972. He received his Ph.D. in Computer Science from the University of ErlangenNürnberg in 2008. His focus of research includes measurement, modelling and simulation of distributed systems as well as deterministic and stochastic Network Calculus. He is currently working as a postdoctoral researcher at the Department of Computer Science (Computer Networks and Communication Systems) at the University of ErlangenNürnberg.

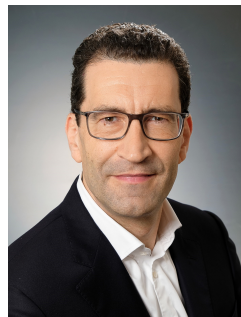

Reinhard German received his master degree in computer science in 1991 and the $\mathrm{PhD}$ degree in 1994 from the Computer Science Department, Technical University of Berlin, Germany. He is a Full Professor at the Computer Networks Lab in the Department of Computer Science, University Erlangen-Nürnberg, Germany. He is also an Adjunct Professor at the Faculty of Information Technology of Monash University, Melbourne, Australia. His research interests include performance and dependability analysis of interconnected systems based on numerical analysis, network calculus, discrete-event simulation, measurements, and testing. Vehicular communications and smart energy constitute major application domains. 